\title{
A quantum theory of disease, including cancer and aging
}

\author{
Jerry I. Jacobson * \\ Institute of Theoretical Physics and Advanced Studies for Biophysical Research, Jupiter, FL, USA
}

\begin{abstract}
A unique, non-invasive, holistic paradigm is presented for amelioration of aging and associated pathophysiologic conditions based upon biological piezoelectricity. The capacity for biostructures to amplify weak electromagnetic (EM) triggers through photon-phonon transductions may permit target-specific magnetic resonance energies to renormalize structural and functional integrity of critical biomolecules, such as telomeres, neurotransmitters, immunogenic transmissible microbiological agents, enzymes, peptide hormone trophic factors, transforming DNA segments... etc. Amelioration of the aging process and the effects thereof, including potentialities for palliation of a diversity of conditions may be possible, utilizing nonionizing physiologic PicoTesla electromagnetic fields. Various quantum phenomena are hypothesized as underpinning mechanisms for both normal and diseased conditions, including piezoelectricity, cyclotron resonance, the Quantum Hall Effect, and magnetic resonance; leading to a discussion of biological fission, fusion, and super conduction in neoplasia. A new particle-wave equation is presented for calculating bio-interaction energies set in dual resonance with intrinsic energies of target masses, to induce quantum transpositional states therein restoring biological order; with concomitant reduction of immunogenic quantum state entropy in selective magnetic domains. Experimental studies demonstrating fundamental positive changes in biological models are discussed in support of this non-significant risk methodology. Such changes include electromagnetic modulation of autonomic nervous system (ANS) tonicity, regeneration of nerve ultrastructure in concert with restoration of functionality, diminished viability and/or proliferation rate in human mammary carcinoma cells, and positive clinical studies in neurological disorders such as Parkinson's disease. Indeed, it appears that a new frontier in biology and medicine is being manifested through advances in electrophysiology, promising hope for palliation of the human condition in aging and disease.
\end{abstract}

\section{Introduction}

This paper examines the possible underpinning mechanisms of disease from an atomistic/molecular perspective. The focus is placed upon the electromagnetic nature of life, and its relation to fundamental aspects of physiology and the maintenance of homeostasis. Normal aging processes are differentiated from various states of entropy, especially neoplasia, in consideration of quantum phenomenological mechanisms. Such mechanisms include: cyclotron resonance, Zeeman resonance, the Quantum Hall Effect, the piezoelectric effect, and Jacobson Resonance. The ultimate aim of the analysis rests in the proposition that externally sourced non-ionizing electromagnetic fields (EMFs) may restore intrinsic electromagnetic profiles of tissues and organs. More specifically, it is proposed that PicoTesla range EMFs are physiologic, and through photon/phonon transductions, i.e. the piezoelectric effect, may restore biologic order. Target specific resonance energies are therein calculable utilizing the formula $\mathrm{mc}^{2}=\mathrm{BvLq}$ (Jacobson Resonance) for magnetic field amplitude, and $\mathrm{f}=\mathrm{qb} / 2 \pi \mathrm{m}$ (cyclotron resonance) is used to establish frequency correlations. Thus, intrinsic molecular energies are set in dual resonance with electromagnetic interaction energies, i.e. wave energies produced by interacting the whole body with the magnetic signals. Numerous basic science and clinical studies are referenced in support of the methodology described. Finally, a particular approach to ameliorating the normal aging process, as well as cancer, is provided by utilizing the method described. In so doing, we may establish bio resonant regulation of molecular intercommunications and cooperation, most especially for telomeres and telomerase [1-11].

\section{Limits of biological entropic reversibility}

Biological systems are intrinsically ordered universes of biochemical, electromagnetic and gravitational interactions in constant flux. The components of such complex organisms oscillate about steady state systems to maintain global equilibrium. Coherence, cooperativity, and the congruence of oscillatory frequencies and trajectories of biological micro components essential to retain physiologic homeostatic signal transductive coupling mechanisms are maintained through interatomic communications networks. Yet, an imperfect material body always subsists with a certain amount of disorder, inherently in possession of configurational entropy. In consideration of quantum discontinuities and disequilibria inherent in any system, there is configurational entropy e.g. alterations of vital structures such as alpha and beta sheaths of proteins, possibly having piezoelectric uninterrupted reticulum networks. In the human body this can reduce free energy available for ordering ATP that drives the metabolic engine of life [1,12-14].

Generalizing, in a biological reversible process, a system moves from a state of initial equilibrium to a state of final equilibrium, through ongoing sequences of equilibrium states. At any time during the process, the system as a state function remains in thermal and mechanical equilibrium with its surroundings. The direction of such a process can be reversed at any instant by an infinitesimal change in extrinsic conditions. Furthermore, reversibility is an idealization that cannot be realized even from a macroscopic perspective. Therefore, an

Correspondence to: Dr. Jerry I. Jacobson, Professor of Medical Physics, Institute of Theoretical Physics and Advanced Studies for Biophysical Research, 2006 Mainsail Circle, Jupiter FL, 33477, Tel: (561) 746-8719; Fax: (561) 748-8870; E-mail: Drjijacobson@yahoo.com

Key words: aging, piezoelectric effect, the quantum hall effect, cyclotron resonance, jacobson resonance, photon-phonon transduction, cancer

Received: December 06, 2015; Accepted: February 11, 2016; Published: February 15, 2016 
actual process may approach reversibility to the extent that it is quasistatic (e.g. extremely slow), whereby dissipative effects are ostensibly negligible. Thus, the entropic changes of a biological system are always greater than the ideal or reversible value. When a biological system departs from and then returns to an initial state via a real process, e.g. transient enhanced sympathetic innervation, the quantum state entropy must increase even though entropy as a state function does not change. Transpositional quantum state elements result in desiccation of microstructures, and disequilibria in small volumes of tissues, secondary to incoherent interatomic messaging that inexorably follows. Incongruous disharmony of molecules and molecular assemblies then permit (1) genetic information transfer mechanisms produce errors, and (2) immunogenic transmissible microbiological agents may then utilize radiative photonic energies that are needed to maintain/restore quantum state normalcy [1,15].

More particularly, any thermodynamic system has a state function $S$, or entropy, which is defined:

$$
d S=d q / T
$$

Where $\mathrm{T}$ is the Kelvin temperature, dq is the change in heat energy, and $\mathrm{dS}$ is the change in entropy, and the Clausius [15,16] equation $\mathrm{d} S=d q / T$ holds for biological systems (reversible processes). Since $\mathrm{S}$ is a state function, the change in entropy accompanying a reversible process connecting the initial and final states can be calculated by integrating $d q / T$ along a path of a reversible process. The relevance of the entropy function is based upon the second law of thermodynamics. In any process, the total entropy of a system and its surroundings increases, yet in a reversible process the state function does not change. The second law of thermo dynamics applies to the system alone when the system is isolated, i.e. the system in no way interacts with the surrounding environment. This means that a biological system, defined as a reversible system does not change macroscopically, as a state function, but does change slowly on a quantum level in small volumes of tissue spaces, via normal aging processes. Because, reversibility is an idealization and infinitesimal changes must occur naturally through time, especially due to changes in the environment, which produce various stressors [1].

Finally, as time-dependent microscopic changes increase quantum state entropy, this permits interactions that further denigrate critical cooperativity of systems dependent upon coherence of signal transduction coupling mechanisms. Thus, the capacity for reversibility of fundamental structural/functional domains dependent ultimately upon interatomic communications networks, diminishes, moving the organism to a state function of irreversibility such as cancer. With increasing failure of genetic information transfer mechanisms, the organism becomes more susceptible to disease, e.g. increasing vulnerability to RNA and DNA transmissible immunogenic agents. Reversibility as a state function of the whole organism ultimately decreases to therein manifest macroscopic degeneration and cessation of function $[1,3,6,15,16]$.

\section{The piezoelectric effect in biology and medicine}

Throughout the ages, sound energy was used only in the process of heating. Pierre Curie $[13,17,18]$ then discovered an amazing property of quartz that inevitably led to many new applications of sound. Curie's discovery is known as the piezoelectric effect. Imagine, for example, a quartz crystal sandwiched between metal plates. When the plates are connected to a source of alternating voltage, having a particular frequency, the quartz crystal will vibrate mechanically at the same frequency. The semi conductive quartz crystal changes alternating electrical energy, i.e. electromagnetic oscillations, into vibrational or mechanical energy. This effect is known as electromechanical transduction. Thus, when certain materials are subjected to mechanical stress, an electrical polarization is set up in the crystal, wherein the crystalline faces become electrically charged. The polarity of the charges is reversible when the compression is changed to tension. Conversely, when an electric field is applied across the material, there is an associated expansion or contraction in accord with the sign of the electric field. A biological analog can be extrapolated in that the diameters of collagen fibers in tendons increase with the growth of a maturing animal. That is, the collagen fibers reflect the tensions to which they are subjected. When a tendon is severed, it is known that DNA synthesis increases in the first day within the peritendinous sheath. Neuromagnetic measurements of responses to auditory stimuli (pure tones) have been used to deduce the location of cortical activity. The evoked field source increases in depth beneath the scalp with increasing frequency of the tone. Assuming that the active region of the primary auditory cortex has uniform width and density of neurons, the observed tonotopic logarithmic mapping implies that the same number of neurons in the cortex is dedicated to each octave in frequency span. This shows the conversion of mechanical energy to electromagnetic energy by human tissue [19]. Marino et al. [20] established a link between bound surface charge distribution and ionic current in permeating interstitial fluid of bone. They recognized the possibility that the piezoelectric signal may have altered the chemical nature of important macromolecules such as collagen, and cellular activity may have been directly influenced [19-24]. Piezoelectric elements that convert mechanical vibrations to electromagnetic oscillations include crystal microphones, the piezoelectric stethoscope, ultrasonic surgical procedures, and underwater hydrophones. The piezoelectric crystal is utilized to generate an electromotive force when subjected to mechanical vibration or sound, and the field of ultrasonics is based upon the piezoelectric effect. Generalizing, when the atomic crystal lattice structure of semiconductors is subjected to mechanical pressure or temperature alterations, a charge displacement occurs on the crystalline faces resulting in a potential difference between the faces. Conversely, when an electromotive force is applied between the crystalline faces of semiconductors, the crystals become dimensionally deformed [13,19-28].

It is of special interest to note Bjorn Nordenstrom's theory concerning biologically closed electric circuits. Nordenstrom considered that the resistivity of human tissue falls within the range of $80-120 \mathrm{ohms}$ per centimeter. Furthermore, Nordenstrom pointed out that the resistivity of capillary walls is 200-300 times greater than that of blood plasma. This means that we may possibly see a direct numerical correlation to $\mathrm{h} / \mathrm{e}^{2}(25,813 \mathrm{ohms})$ the flux quantum as it may relate to the fine structure constant. This extrapolation is based upon the resistivity of Nordenstrom's proposed "Insulator cables" (capillary walls) which comprise the capacitive factor of his hypothesis, "vascular interstitial closed circuits;" a system he proposed to connect cancerous lesions to the surrounding tissues electrically $[1,19,29]$.

The piezoelectric effect is observed in all ferromagnetic and nonferromagnetic crystals that reveal a symmetry and have one or more polar axes. And, the magnitude of the piezoelectric effect depends on the direction of the applied stress relative to the crystal axes. Now, biologists and biophysicists are focusing attention upon intracellular and extracellular filamentous structures, because these polymorphic complex structures are involved in metabolic controls and not merely 
in mechanical structure. Filamentous structures may be viewed in a new light, considering (1) current knowledge concerning photo and infraoptical emissions of biosystems, (2) connections between ordered electron and protonic flows associated with semiconductor biostructures, (3) protein structure, e.g. filament shaped assemblies of globular proteins, especially alpha helices and beta sheaths and (4) H-bond reticulum [13,19,29-32].

Microtubules (MT) contain tubulin dimers that may act as dipoles, since alpha tubulin is negatively charged and oriented towards the cellular center, whereas beta tubulin is positively charged and outwardly oriented. The bioelectric structure of MT's reveals an ordered structure with oriented dipoles, and are considered electrets; characterized by piezoelectric activity, involved in photon-phonon conversion, and transmission of quantized electromagnetic and mechanical perturbations [32].

Piezoelectric mechanisms may be spontaneously present in human physiology. For example, in specialized cells involving reception of external stimuli (heat, pressure and sound), such cells may convert the special types of energy they are sensitive to, into the electrical energy of a nervous impulse [1]. Bistolfi [31-33] reported that proteins containing more than one alpha helix have a nearly spherical polyhedral geometry, whereas such structures imbue quesi-crystalline characteristics to suggest piezoelectric activity. Franco Bistolfi [31] maintained, "Alpha helices can be compared to piezoelectric polypeptide springs able to transform chemical and electric energy into mechanical energy, and then back into electric energy." Bistolfi further informs that the piezoelectric effect may be responsible for vascular effects, actions of smooth muscles and effects on fibrous connective tissues, in full accord with Nordenstrom's theory of vascular interstitial closed circuits. Piezoelectricity may also serve to explain reactivation of quiescent cells and proliferative stimuli involving photon-phonon transductions related to alpha helices, microtubules and DNA. Such triggers are also hypothesized to be involved with cytoplasmic and membrane signaling. Teller [13,34] has stated, "Energy must be present in an ordered form to carry out a given job: this happens when within a certain space or volume, most particles move in the same direction." Such is the case when phonons are induced within magnetic domains, such as telomeres; leaving open the possibility for calculable dual resonance models that equate intrinsic energies of target masses and electromagnetic interaction energies. This may provide structural integrity to telomeres for amelioration of the aging process, among numerous other potentialities [1].

The inward stream of signals into cells is the result of a complex sequence, beginning with binding of humoral molecules that provide stimulation at their specialized binding sites. A rippling effect is elicited extending along the membrane's outer layer. Altered calcium binding to glycoproteins affects membrane related proteins serving receptor and enzymatic functions. When a weak electrical or chemical stimulus is sensed, signals are transmitted to the cell's interior along coupling proteins, and this interaction may include non-linear vibrational waves (solitons) in the amide spines of helical proteins. These intramembranous proteins (IMP's) span the membrane from surfaces to cellular interiors. There is additionally an outward stream of signals at cellular membranes, and it mediates the organization of the cell surface in "allogenic cytotoxicity," seen in lymphocytes targeted against tumor cells [35-37].

Now, we consider the conundrum: What is the underlying physical mechanism by which non-ionizing, extremely low-frequency, electromagnetic fields, (EMF's) affect biosystems? Researchers have rejected the idea that the interaction can be based upon the non-ionizing energy of a single photonic interaction, concluding that a biological effect must therefore be integrated over time. We herein propose a significantly different explanation. We may accept the hydrophobicity of IMP H-bonds that cross the phospholipid bilayer of membranes; and the necessity for photonic recycling in cell surface interactions after dissipation of energies. Present models have lacked structure and thermodynamic properties to maintain sufficient energies for amplifications by a factor of $10^{12}$. We suggest that when the ligandreceptor association alters conformational states of extruding portions of IMP's, non-linear vibrational waves (solitary solutions to non-linear relativistic field problems) result from photon-phonon transductions/ conversions, representing the piezoelectric effect. However, when the EM interaction energy is equal to the intrinsic energy of a critical molecule, the result is a direct enhancement of the vibrational energy of the molecule. In other words, there is another kind of interaction occurring, not dependent upon the single photon interacting at the membrane surface. The resultant affectation is formulated to be a gravity wave acting directly upon the molecule. This hypothesis does not discount the effects of interactions directed from single photonic interactions at membrane surfaces, which promulgate solitons. EM oscillations are directly converted to mechanical vibrations by the single molecule / molecular assembly. Indeed, the H-bonds of singular relevance are those of proteins having vibrational frequencies about $10^{12}$ and DNA whose vibrational frequencies are $10^{11}$. These critically important H-bonds may be viewed as centers of EM radiation in the range from the millimeter microwave to the far infrared. Classical electro dynamical theory does not yield a model for biomolecular dual resonance responsiveness, but Jacobson Resonance theory does accomplish this task by providing a physico- mathematical apparatus that is empirically testable [12,27,31,35-45].

Equivalencies have been derived showing Jacobson Resonance as a generic expression for Zeeman Resonance (zero-order magnetic resonance) and cyclotron resonance $[12,19,46]$.

The mathematical fusion of the equations of quantum theory and special relativity yielded a quantum field theory fraught with niggling infinities only removable through the process of renormalization, quite unsatisfactorily. Fusion of quantum field theory with general relativity has only been accomplished with superstring theory, a complicated abstruse mathematical exercise with difficulty in testing empirically. The search is basically for a theory that explains quantum gravity that can be verified empirically [38].

We note that quantum mechanics affords statements relating to ostensible discontinuous transitions from one total system to another. It does not afford a representation of the specific process. This relates to the idea that quantum mechanics does not operate with single systems, but with a totality of systems. Considering this interpretation of quantum mechanics, it is quite clear why this theory does account for the fact that weak disturbing forces are capable of providing alterations of any magnitude in the physical condition of a system. Such forces provide interference patterns of electro-gravitational circuits in spacetime, wherein only very small alterations of the statistical density in the ensemble of systems occur. Hence, infinitely weak alterations of single systems, e.g. PTEMF interactions with biosystems, are required for profound amplifications within complex systems. Our analysis may provide a new method by which one may acquire a glimpse of the nature of critical alterations of the single system. Indeed, in our discussion of biological entropy, it was noted that the environment 
is critically important to maintain a reversible system, i.e. in that infinitesimal changes (intrinsic/extrinsic) can make the difference for retaining homeostatic function. The hidden variable that is required to make sense of the disparate elements in both general relativity and quantum theory may be the biological model. One issue for us to consider is the notion in quantum theory that quantum fluctuations can give rise to the appearance of "virtual particles" out of nothing (the vacuum), provided that the particles mutually annihilate before their existence violates the uncertainty principle. It is this author's belief that virtual photon flux is the basis for photon-phonon transductions in biosystems, yielding a quantum gravitational field/ physiologic flux densities in the PT range and lower- down to an attogauss $[13,47,48]$.

In Barbara McClintock's system, [49] the controlling elements of genes do not correspond to stable loci on the chromosome- they move. In fact, transposition is itself a property that can be controlled by regulator or activator genes. Not only can viral DNA insert itself into the DNA of a host cell, and thereafter detach itself, but the normal DNA of a cell can rearrange itself. This regulation is subject to influence by the environment of the cell. Information flow is not unidirectional, but flows to and from genes that vibrate in space, and the vibrations are regulable by elements of the organism essential to the root of life itself. Researchers have catalogued numerous chromosomal alterations that appear in association with certain cancers. Logical connections have been made between oncogenes, discreet transforming DNA segments (about 350-1,000 base pairs), translocations, deletions, and other much studied chromosomal changes. An example is the myc oncogene, moving from a normal position in chromosome 8 to a region of chromosome 14, landing close to one of the genes for immunoglobulins. Proteins are subject to electrophoresis in magnetic fields. Since DNA is subject to mechanical influence by mRNA and mRNA is subject to influence by proteins, the directionality of information flow is to and from genes and is subject to magnetic fields. Genes continually change structurally throughout the life cycle as demonstrated by Susumu Tonegawa $[19,50]$ while outlining the genetic principle for generation of antibody diversity. As such, we denote the interplay between various potential target elements, subject to incessant adjustment mechanisms: electromagnetic/ biochemical. Polypeptide trophic factor and oncogene research converged when homology between some oncogenes and growth factors or their receptors was shown by sequence analysis. Evidence has markedly increased in that excessive synthesis or an altered version of polypeptide growth factors (or of their receptors) may result in transformation of recipient cells $[1,19,51]$.

Certain oncogene products can induce differentiation of recipient cells. This calls attention to another facet of the intricate interplay between differentiative as well as transforming processes. The case of $\mathrm{H}$-ras and that of $\mathrm{V}$-src, whose expression in PC12 cells results in mitotic arrest and neuronal differentiation comparable to those elicited by NGF, provide instances of a growing list. Support for the involvement of cellular oncogene products in the normal physiological regulation of cell growth comes also from the evidence that peptide hormone growth factors share a common mechanism of action with viral oncogene protein products [52].

Rita Levi Montalcini [53] maintained that whenever cell death of specific neuronal populations is connected to the availability of neurotropic factors such as NGF, its exogenous supply or endogenous production may offer a promise and approach to presently incurable diseases [54]. Cancer is no longer considered more than 100 diseases, each of them characterized by a different tumor type heterogeneous on a cellular level. Instead there are a small number of molecular mechanisms which manifest common denominators to all tumor types. Indeed, an understanding of the functions of oncogene proteins makes it possible to develop a therapy designed to strike at the central defects of the cancer cells [21].

Therefore, we note relevant relations between the transforming DNA in human cancers, and oncogene related proteins, trophic factors and viral nucleic acids in that these piezoelectric crystalline structures may be considered etiological factors which render them targets possibly susceptible to the EM force. Wherein the atomic crystal lattice structures or magnetic domains may be reoriented into normal, physiological spatial positions $[51,52,54,55]$.

Now, we point to a remarkable example of biological piezoelectricity. One of the electrical responses of the cochlea to sound is known as the "cochlear microphonic," a potential fluctuation recorded between an electrode on or near the cochlea, and an indifferent electrode. This is generated by deformation of the processes of the hair cells, and is linearly proportional to the magnitude of the displacement of the basement membrane. Consequentially, it reproduces the waveform of sound stimulus. The reproduction of the frequency and the magnitude of the sound is so accurate that if music is played into an animal's ear, a faithful rendition of the song may be produced by feeding the cochlear micro phonic into an audio amplifier. The cochlea microphonic can be recorded from the auditory nerve close to the cochlea, but it is not a neural response. Particularly, it can be obtained from a recently killed animal in which neural activity has ceased. Clearly, it is produced by mechanical distortion of the hair cells exemplifying the piezoelectric effect in biological tissue [19]. The basis of aging and disease is derived from varying transpositional elemental quantum entropic states, with production of biological disorder $[1,13]$. Firstly, aging processes are affected by intrinsic and extrinsic factors. Intrinsic factors involve inherited genomic predispositional factors such as telomere length regulating the maximal number of cell divisions that might be remaining in a tissue/organ before senescence sets in. Extrinsic factors include environmental stressors, generally increasing HPA axis stimulation and production of cortisol, the primary effect of which is the enhancement of gluconeogenesis to meet energy exigencies, i.e. especially for the brain and major muscles. Yet, the greater the production of heat energies, the faster a progressive burn occurs, increasing quantum entropy, microscopic scarring in small volumes of tissues, desiccation and aging (quantum state, biologically disordered immunogenic domains). Interestingly, the basis of disease is not far removed from that of normal aging, because disease represents incurrence of elemental transpositional quantum state entropy until such time that biological order and coherence are affected; such that pathophysiological manifestations are promulgated, e.g. such as thermonuclear fissions/fusions in cancer cells; which may represent an irreversible process. Since biosystems are universes of electromagnetic and electrogravitational activity in constant flux about steady state systems (reversible processes) to maintain equilibrium, we note that reversibility is an idealization and is never realized in real time. Thus quantum state entropy will progressively increase with infinitesimal steps in normal aging, but when cells experience crises stages (particularly M2) reversibility as a state function may well be lost, and loss of function in a particular tissue or in the whole body may result. The ultimate question then remains, "When immunogenic quantum state microbial agents, or microscopic entropic states represent pathophysiological electromagnetic domains, is it possible to reverse incoherencies, biological disorder and disequilibria through a non-invasive holistic paradigm to electromagnetically restore quantum 
biological order; using physiologic PicoTesla range magnetic fields that are target specific?" [1].

\section{Physiologic magnetic field profiles}

Physiologic magnetic profiles of human tissues have been measured directly with superconducting quantum interference detectors, or atomic magnetometers. These magnetic fields have been found to be in the Pico Tesla range. One Pico Tesla is fifty million times weaker than the geomagnetic field (the Earth's steady magnetic field). The existence of the brain's magnetic field and the difference between the magnetic field profile of the normal brain vs. the pathologic brain, has been known from the classical work of David Cohen [56] and several recent works of others using the superconducting quantum interference detectors (SQUID); on patients with epilepsy and other neurologic disorders. These investigators measured the intensity profile of the human brain magnetic field and found that it is on the order of $0.5 \mathrm{PT}$ (PicoTesla) or $5 \times 10^{-9}$ gauss for alpha brain waves [57]. Cohen [56] found that a DC magnetic field on the order of $5 \times 10^{-8}$ gauss (5PT) was associated with delta brain waves, and a maximum measurement of the human heart magnetic field profile was on the order of $5 \times 10^{-7}$ gauss (50PT) [58]. Anninos [59] found that a maximum magnetic field profile for the human brain was about one micro gauss (100PT). Therefore, from a practical point of view, the PicoTesla range of current interest is from about 0.5 PT to about 100 PT. Jacobson Resonance calculations point to even more subtle energies for sustenance of life and potential amelioration of disease [56-60].

It has been hypothesized that physiologic homeostatic mechanisms of living systems operate fundamentally on atomic levels; thus promulgating the idea of an EM immunotherapeutic response. Therefore, it is proposed that physical adjustment mechanisms maintain the balance of total systems in accordance with quantum theory. Recapitulating, quantum mechanics affords statements relating to apparently discontinuous transitions from one total condition to another without yielding a representation of the specific process. This is connected to the notion that this theory does not operate with the single system, but with a totality of systems. With this interpretation of quantum mechanics, it is readily ostensible why this theory accounts for the idea that weak disturbing forces are able to provide alterations of any magnitude in the physical condition of a system, such that only small alterations of the statistical density in the ensemble of systems occurs. Hence, only infinitely weak alterations of single systems, e.g. Pico Tesla magnetic field signals, are required for profound amplifications within complex systems, e.g. biological systems. It appears that a disturbance of the physical parameters of the flow of electrons (mass/ energy) whatever the cause, produces a change in the oscillation frequency of the electronic bioplasma, and thus disturbs the processes of biosystems [13]. It is thought that the adjustment mechanisms that maintain ordered states of matter are based upon photon-phonon transductions or conversions, i.e. the piezoelectric effect. In addition to the idea of aspecific effects provided by administration of NIR's to living systems, Jacobson Resonance theory proposes that target specific signal parameters may be calculable with $\mathrm{Mc}^{2}=\mathrm{BvLq}$; setting in dual resonance the intrinsic energy of a target mass and the electromagnetic energy (BvLq), provided by PicoTesla range magnetic flux densities exogenously sourced $[1,12,16]$.

\section{Supporting literature}

Pico-Tesla electromagnetic fields. (PTEMFs) have been demonstrated to affect brain waves and enhance regeneration of nerve ultrastructure, [12,61] affect autonomic nervous system tonicity, e.g. enhance parasympathetic stimulation to cardiac inputs and regulate atrio-ventricular conduction mechanisms of the heart (affecting rate and rhythmicity) $[9,10]$, modulate endogenous opioid activity (e.g. enkephalin, endorphin), [62] and affect benefits in neurological disorders such as Parkinson's disease, multiple sclerosis, and epilepsy [57-59,63-66] speed wound healing, and regulate thoracic spinal neuronal potentials after administration of noxious chemicals to the heart (which stimulated nociceptive afferent fibers, $[67,68]$ just to cite a few of the many studies conducted at major universities. Indeed, research over the past 30 years has revealed more and more that extrinsically sourced, low-level, extremely low frequency electromagnetic fields (many orders of magnitude weaker than the membrane potential gradient in the pericellular fluid) do modulate actions of hormones, antibodies and neurotransmitter molecules at cell surface receptor sites. The observed sensitivities are as low as $10^{-7}$ volts per centimeter in the extremely low frequency spectrum. [35,36]

Other examples of subtle field effects also include: altered rate of cell growth, [69] suppression of T-lymphocyte cytotoxicity, [70] increases in the growth related enzyme ornithine decarboxylase, [71] altered quantities of RNA transcripts and proteins, [72-78] altered cell surface properties, and effects on development. [35,36]

\section{Photons and phonons in dual resonance}

The particle-wave equation of Jacobson Resonance has yielded basis for Inertial Electromagnetic Induction (IEMI), connecting the phonon field and photons. It explains the associated conjunction of natural phenomena through an algebraic description of the causal nexus.

$$
\frac{\overrightarrow{m_{0} c} \square c}{\sqrt{1-\frac{v^{2}}{c^{2}}}}=(\overrightarrow{\mathrm{B}} x \overrightarrow{\mathrm{V}}) \square \overrightarrow{\mathrm{L}}\left(\mathrm{q}_{\mathrm{J}}\right)
$$

$\mathrm{Mc}^{2}=\mathrm{BvLq}$ (Jacobson Resonance) $[1,12,39,51,61]$ is used to establish the magnetic flux density (B) of an externally applied magnetic field to the whole organism, having longest dimension (L). $\left(\mathrm{mc}^{2}\right)$ represents the intrinsic energy of a target mass $(\mathrm{m})$ which can be any atomic or molecular species, e.g. peptide hormone trophic factor, enzyme, neurotransmitter, DNA structure such as a telomere (TTAGGG), or any immunogenic particle. (BvLq) represents the electromagnetic interaction energy (wave energy), wherein the body of length $(\mathrm{L})$ containing the mass $(\mathrm{m})$ interacts with the magnetic field (B) to provide a system of dual resonance, i.e. $\mathrm{mc}^{2}=\mathrm{BvLq}$. It is hypothesized that a coherent excitation is produced in the target mass $(\mathrm{m})$ via a photon-phonon transduction or conversion, i.e. the piezoelectric effect. (v) May be any inertial velocity such as Earth orbital velocity, because Newton's second law of motion does not distinguish between terrestrial and celestial velocity; and, (q) represents a unit electrical charge, or a single ab-coulomb in the CGS system of physical units; established by defining electromotive force as energy per unit charge. The appearance of $\sqrt{\left(1-v^{2} / c^{2}\right)}$ represents the Einsteinian adjustment for mass, but is negligible for experimental conditions [78].

A detailed description of this physico-mathematical model is available in the literature; including the derivation, rationale for the variables $\mathrm{m}, \mathrm{B}, \mathrm{v}, \mathrm{L}$ and constants $\mathrm{c}$ and $\mathrm{q}$; and the correlation to other known resonance phenomena: ion cyclotron resonance and Zeeman resonance. Whereas, (c) is the velocity of light, and also the velocity of the force carrier (photon) for a magnetic field moving independently of 
the inertial frame of reference/source $[1,12,19]$.

Within the context of this presentation sample calculations follow to show how the theory may be applied to biological systems. After the magnetic flux density (B) is derived from $\mathrm{mc}^{2}=\mathrm{BvLq}$, the derived (B) field is inserted into the ion cyclotron resonance equation, $f=q B / 2 \pi m$, to derive the associated frequency [12].

\section{Sample supporting studies in parkinson's disease}

A clinical pilot study in PD with subjects experiencing motor fluctuations was conducted to determine safety and tolerability with fine tuning of treatment parameters; to ascertain the appropriate PTEMF protocol. Thirteen subjects ( 9 women, 4 men) with an average age of $62+/-13$ years and PD duration of $11.7+/-7.4$ years completed the study. Treatment sessions lasting 1 hour and 20 minutes were performed $3 \mathrm{x} /$ week for a minimum of 3 to 5 weeks. No study related adverse effects were noted, and all subject demonstrated improvement in multiple areas of evaluation in this pilot study. In addition, anecdotal improvements were reported by multiple subjects in the following areas: decreased fatigue, improvement in the quality and quantity of sleep, enhanced sense of smell, increase in the "on" time, and ability to return and/or increase participation level in activities, e.g. golf and driving.

A single center, double blind, randomized, placebo controlled trial utilizing PTEMF as an adjuvant to standard medical therapy in PD patients with motor fluctuations was performed in 12 subjects (6 per group). 24 sessions of 1.5 hour of total body PTEMF were administered over 8 weeks. Standardized motor and non-motor assessments were performed prior to treatment, at endpoint, and monthly for 3 months. The treatment group demonstrated significant improvement over placebo after 8 weeks of therapy. Importantly, improvement on several scales persisted up to 2 months post treatment. No treatment related adverse events were reported. The conclusion: low-level PTEMF may improve motor and non-motor features of PD beyond that achieved with standard medical therapy. These effects are long lasting. Larger placebo-controlled studies are indicated to confirm and further investigate the benefit of this unique, noninvasive and potentially promising therapy.

The signs and symptoms of PD result from widespread neuronal degeneration, resulting in downstream cortical and subcortical dysfunction. We postulate that the beneficial effect of PTEMF results from its piezoelectric effects.

As a result, synaptic activity in neurons at various levels of dysfunctional cortical-basal ganglionic loops in PD might be favorably affected. Further research will obviously be needed to clarify this mechanism of action $[63,79]$.

\section{Sample calculation}

Nerve growth factor (NGF) exhibits trophic influences on a variety of neuronal populations; promoting survivability, regulation of synaptic transmissions, and plasticity at adult synapses in many regions of the central nervous system; and homeostatic regulation of intrinsic neuronal excitability. NGF contains an anti-apoptosis inducing segment to prevent cell death. Choosing NGF as a target, we consider the following:

(1) NGF is 26,500 Dalton, or $4.425 \times 10^{-20}$ gram

(2) $\mathrm{C}^{2}=9 \times 10^{20} \mathrm{~cm}^{2} \mathrm{sec}^{-2}$
(3) (L) is the height of a human, or $177 \mathrm{~cm}$.

(4) (V) is Earth orbital velocity, or $3 \times 10^{6} \mathrm{~cm} \mathrm{sec}^{-1}$

(5) (q) is one ab-coulomb (unit charge by definition)

The CGS system of physical units is chosen, because, in the MKS (SI) system force is determined between moving charges, whereas in the CGS system force is determined between stationary charges. Therefore, we desire:

$$
\begin{aligned}
& \mathrm{Mc}^{2}=\mathrm{BvLq} \\
& \left(4.425 \times 10^{-20} \mathrm{gm}\right)\left(9 \times 10^{20} \mathrm{~cm}^{2} \mathrm{sec}^{-2}\right)= \\
& \left(7.5 \times 10^{-8} \text { Gauss }\right)\left(3 \times 10^{6} \mathrm{~cm} \mathrm{sec}^{-1}\right)(177 \mathrm{~cm})(\text { ab-coulomb })
\end{aligned}
$$

Then, we note that (q) is normalized in CGS. Consequently,, when converting from CGS to MKS, $\mathrm{mc}^{2}=$ BvLq becomes $\mathrm{mc}^{2}=\mathrm{BvL}$ (10q), because $1 \mathrm{ab}$-coulomb is equal to 10 coulomb. Therefore, when using the MKS expression, $f=q B / 2 \pi m$, we must use $f=10 q B / 2 \pi m$, and we note:

$$
\begin{aligned}
\mathrm{f} & =\frac{10\left(1.6 \times 10^{-20} a b-\text { coul }\right)\left(7.5 \times 10^{-8} \text { Gauss }\right)}{(6.2832)\left(9.11 \times 10^{-28} \mathrm{gm}\right)} \\
& =2.1 \mathrm{~Hz}
\end{aligned}
$$

Where, $(\mathrm{q})$ is the charge of an electron and ( $\mathrm{m})$ is its mass.

Normalization permits the process of introducing a numerical factor into an equation and is of importance in quantum mechanics. Furthermore, the signal, 7.5 Pico-Tesla @2.1 Hz, has been successfully utilized in the treatment of Parkinson's disease, improving the quality of life for these patients. [1,63].

\section{Cardioelectromagnetic}

\section{Heart rate variability (HRV)}

A human heart rate varies with every heartbeat, through electro physiologic adjustments to maintain homeostasis. Our bodies are a collection of trillions of atoms in mutual cooperation creating reversible processes such that all the interactions oscillate about a steady state system to regulate homeostatic function. Remarkably, any change to the body, e.g. stressor, is automatically reflected in each heartbeat. Heart rate variability represents the variation of beat-to-beat intervals, i.e. $\mathrm{R}-\mathrm{R}$ intervals (Figure 1).

\section{Review of experimental studies}

In accord with the diversity of possibilities presented herein, concerning biological effects secondary to application of non-ionizing extremely low intensity and low frequency EMF's, we point to studies conducted at the University of Oklahoma Health Sciences Center, Arrhythmia Research Institute.

It appears that a distinctive, and quite unique potential is unfolding

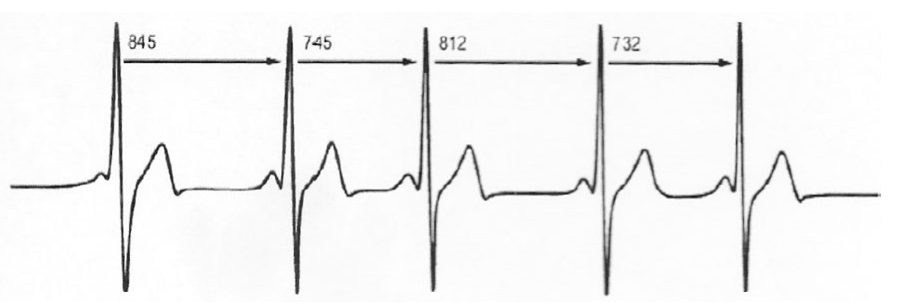

Figure 1. Heart Rate Variability. 
from the electro physiologic sciences for ameliorating the aging process and the effects therefrom.

In our initial experimental study we used 2 different sized Helmholtz coils to apply micro Gauss $(\mu \mathrm{G})$ levels of electromagnetic fields (EMFs) either to the vagosympathetic trunks or across the chest of anesthetized dogs. From previous reports on frequency analysis of heart rate variability, the sympathetic activity averaged $0.043 \mathrm{~Hz}$. Using the Jacobson $\left(m c^{2}=q_{J} v B L\right)$ and Cyclotron Resonance $\left(f=\frac{q b}{2 \pi m}\right)$ equations, we calculated the correspondent EMF amplitude value of $2.87 \times 10^{-6}$ Gauss. Applying these EMFs at the vagal trunks invasively or across the chest non-invasively, we found enhanced parasympathetic effects on the heart rate and atrioventricular conduction (AVC), both properties influenced by parasympathetic innervation. The maximal heart rate change in the experimental versus control groups was $29 \%$ versus $12 \%(\mathrm{P}=0.03)$. The same EMF stimulation decreased the voltage applied to the vagal trunks by $60 \%$ in the experimental group versus a $5 \%$ increase in the control group $(\mathrm{P}=0.005)$. We note the right and left femoral veins were cannulated for delivery of fluids and anesthetics, and for the insertion of an electrode catheter which was advanced and positioned against the lateral atrial wall in the low right atrium for atrial pacing. Using another level of EMF, (amplitude, $0.34 \mu \mathrm{G}$ and 2 $\mathrm{kHz}$ ) determined empirically, applied as above, there was a significant increase in atrial arrhythmias, including atrial fibrillation (AF) atrial premature depolarization, and atrial tachycardia, which could be suppressed by applied EMF, $(2.87 \mu \mathrm{G}$ at $0.043 \mathrm{~Hz})$. It should be pointed out that $2 \mathrm{kHz}$ is a non-physiologic frequency and 0.34 micro gauss is sympathomimetic.

A shortcoming of these studies was the lack of a mechanism underlying these responses to low level EMFs [10]. Subsequently, a series of experimental studies have been published [80-84] in which we used low-level vagosympathetic trunk electrical stimulation at levels $10 \%$ and $50 \%$, which did not slow the heart rate or slow atrio ventricular, conduction (AVC). In an experimental model of induced $\mathrm{AF}$, it was found that the nerve clusters called ganglionated plexi (GP) found in specific vulnerable sites in the atria became hyperactive under the influence of excessive release of cholinergic (parasympathetic) and adrenergic (sympathetic) neurotransmitters. In this regard, Smith et al. [85] tested the function of the GP, weeks after separation of the vagal and sympathetic nerves from the aforementioned structures. Not only did the intrinsic GP neurons remain viable but their responsiveness was enhanced. To emphasize this point, we severed the neural connection from the brain to the GP in experimental animals and found after 10 weeks there was a progressive increase in the occurrence of paroxysmal atrial fibrillation. Low-level vagal nerve stimulation markedly attenuated the hyperactive state of the GP, thereby suppressing AF. In a recent experimental study, we recorded the neural activity of the GP and found that several hours of induced AF caused a significant increase in the amplitude and frequency, whereas low level vagal nerve stimulation not only suppressed AF propensity but also the increased amplitude and frequency of the hyperactive GP. A recent clinical report from our group has confirmed that low-level vagal nerve stimulation can mitigate AF in patients with the paroxysmal form of this arrhythmia.

Since electrical stimulation of nerves induces its actions via release of chemicals called neurotransmitters, we found that a specific peptide, vasostatin-1 was released at low-levels of vagal nerve stimulation (50\% below the voltage that causes slowing of the heart rate) and even at very low levels of vagal nerve stimulation ( $80 \%$ below the slowing threshold).
Indeed, further studies in the experimental model of induced AF, showed that vasostatin-1 suppressed AF by inhibiting GP hyperactivity by an anti-autonomic action mediated by nitric oxide $[86,87]$.

Returning to the earlier studies using low level EMFs to affect heart rate and rhythm, we inserted the molecular weight value for vasostatin-1 into the Jacobson and Cyclotron Resonance equations to derive the amplitude $(0.034 \mu \mathrm{G}$ or $3.4 \mathrm{PT})$ and frequency $(0.952 \mathrm{~Hz})$, respectively. Applying these EMFs at the vagal trunks and across the chest we found that these low level fields significantly suppressed AF and also decreased the amplitude and frequency of the neural activity of the hyperactive GP [88].

A fundamental question is: Why do these neural tissues (GP) on the heart become hyperactive in some of the population, in particular, disproportionately in persons from 60-80 years old? An early report by Kaijser et al. [89] studied healthy women and men comprising groups, age 20-40, 40-60, and those between ages 60-80. Using simple procedures, such as handgrip and the response to the dive reflex test on heart rate and blood pressure, they found, "There seems to be only a moderate attenuation of autonomic cardiovascular responses to about 60 years, after which there is a more rapid decline." Since these cardiovascular responses are mediated by the autonomic innervation from the brain to the heart, these studies suggest that with age the control of the GP on the heart by the higher centers is markedly attenuated allowing these lower centers to become independently hyperactive. This would help to explain the increased incidence of AF in the elderly population compared to younger cohorts [90,91].

Now, although parasympathetic stimulation has been proposed as a generic approach to anti-aging, it must be pointed out that for various indications, e.g. obesity, fibromyalgia, hypertension in cases usually refractory to pharmacological intervention, esophageal reflux, autism, and diabetes...etc., sympathomimetic electromagnetic field intervention may be useful. Understanding physiologic mechanisms underlying various clinical symptomatology is important, and requires extensive ongoing research [91-97]. It should be noted that there are times when the body's physiologic mechanisms will reverse the anticipated effect of a therapeutic signal. Indeed, the intentionality of magnetic resonance therapy is to intervene in such a manner so as to assist the body to heal itself subsequent to the intervention.

Einstein [96] once defined the grand aim of science as, "To cover the greatest number of empirical facts by logical deduction from the smallest possible number of hypotheses or axioms." He believed in a basic universal field in which the multifarious manifestations are merely particular ephemeral forms or conditions of state. Indeed, Lincoln Barnett [92,97] said, "The urge to consolidate premises, to unify concepts, to penetrate the variety and particularity of the manifest world to the undifferentiated unity that lies beyond is not only the leaven of science, it is the loftiest passion of the human intellect."

\section{Clinical perspective}

"Despite the superiority of catheter ablation to drug therapy, recurrence of atrial fibrillation $(\mathrm{AF})$ or atrial tachycardia is still common for patients with paroxysmal AF who underwent AF ablation. The critical study described a noninvasive therapy to treat AF in a canine model of AF. When an extremely low-level electromagnetic field $(0.034$ micro gauss at $0.0952 \mathrm{hertz}$ ) was applied through a pair of Helmholtz coils across the canine chest wall, the atrial effective refractory period was prolonged, AF inducibility was reduced, and the neural activity in the ganglionated plexi was suppressed. The study demonstrates that 
AF can be potentially controlled noninvasively by PTEMF's within 2-3 hours after it was initiated. Early termination of AF by PTEMF's, if proven by clinical studies, will have a significantly positive impact on the use of antiarrhythmic drugs and oral anticoagulation therapy, particularly for patients in the earlier stage of paroxysmal AF. This approach, if effective, has a unique advantage that will not destroy any myocardium and perhaps avoid introducing iatrogenic atrial tachycardia's" [88].

\section{Sample calculation and specific study sample}

Vasostatin-1 was chosen as the target molecule for antiarrhythmogenic studies for its inhibitory effects on the cardiac autonomic nervous system (CANS), particularly the adrenergic component. Vasostatin-1 is a recently recognized cardio regulatory peptide with diverse actions, including anti-adrenergic and antiinflammatory effects. It was shown that vasostatin-1 injection into the major atrial ganglionated plexi (GP) suppressed atrial fibrillation (AF) inducibility and inhibited the activity of the intrinsic CANS. These results indicated that vasostatin-1 mimicked the anti-arrhythmic effects of low-level vagal nerve stimulation ( $\mathrm{LL}=\mathrm{VNS}$ ), supporting the notion that vasostatin-1 may be one of the critical neuromodulators mediating the effects of LL-VNS on AF susceptibility and intrinsic CANS inhibition. Studies were done on adult dogs. Therefore, we consider:

1) Molecular weight of VS- $1=7000$ Dalton or $1.169 \times 10^{-20}$ gram.

2) $\mathrm{L}=100 \mathrm{~cm}$ (length of an adult dog)

3) $\mathrm{C}^{2}=9 \mathrm{X} 10^{20} \mathrm{~cm}^{2} / \mathrm{sec}^{2}$

4) V= Earth orbital velocity $=3 \times 10^{6} \mathrm{~cm} / \mathrm{sex}$

5) $\mathrm{q}=$ one ab-coulomb, by definition. Thus, $\mathrm{mc}^{2}=\mathrm{BvLq}$ :

$\left.\left(1.169 \times 10^{-20} \mathrm{~g}\right) 9 \times 10^{20} \mathrm{~cm}^{2} \mathrm{sec}^{-2}\right)=\left(3.4 \times 10^{-8} \mathrm{Gauss}\right)\left(3 \times 10^{6} \mathrm{cmsec}^{-1}\right)$ $(100 \mathrm{~cm})(1 \mathrm{ab}-\mathrm{C})$

Then, $\mathrm{f}=\mathrm{qB} / 2 \pi \mathrm{m}$ yields:

$0.952 \mathrm{H}_{2}=\frac{1.602 \times 10^{-19} \text { ab-C }\left(3.4 \times 10^{-10} \text { Gauss }\right)}{(6.28)\left(9.1095 \times 10^{-28} \mathrm{~g}\right)}$

The use of low-level PicoTesla electromagnetic fields to suppress atrial fibrillation was studied by $\mathrm{Yu}$ et al. [88] at the University of Oklahoma Health Sciences Center, Heart Rhythm Institute. It was considered that extremely low-level EMF's were proposed to cause significant changes in neural networks, and it was decided that investigation of the effects of PTEMF's on arrhythmias would manifest positive outcomes. The question was asked, "Can PTEMF's suppress atrial fibrillation?" In seventeen dogs anaesthetized with pentobarbital, bi-lateral thoracotomies permitted the placement of multi electrode catheters in both atria and at all pulmonary veins. Atrial fibrillation, (AF) was induced by rapid atrial pacing (RAP) or programmed atrial extra stimulation. At the baseline and at the end of each hour of RAP during sinus rhythm, programed atrial stimulation yielded the effective refractory period (ERP) as well as the width of the window of vulnerability (WOV). WOV was a measure of AF inducibility. Microelectrodes were inserted into the anterior right ganglionated plexi (ARGP) and recorded neural firing. Helmholtz coils were utilized, powered by an HP generator that produced the PTEMF signal parameters shown in the aforementioned calculation, i.e. 0.034 micro Gauss at 0.952 Hertz. The study sample was divided into two groups. Group 1, $(\mathrm{n}=7)$ included application of PTEMF to both cervical vagal trunks. Group 2, $(\mathrm{n}=10)$ included application of PTEMF across the chest so that the heart was located in the center between the coils. In Group 1, PTEMF induced a progressive increase in atrial fibrillation threshold at all pulmonary vein and atrial sites $(\mathrm{p}<0.05)$.

Group 2 revealed an atrial ERP which was progressively shortened; and ERP dispersion and WOV progressively increased $(\mathrm{p}<0.05)$ compared to baseline values during three hours of RAP. Then, the next three hours included combined application of RAP plus PTEMF which increased ERP and decreased the WOV until the end of the third hour. The amplitude and frequency of neural activity recorded from the ARGP were significantly suppressed by PTEMF in Groups 1 and 2 [9].

\section{Gerotargets and magnetic resonance energies}

A new non-invasive holistic paradigm has been proposed for ameliorating the aging process and the effects therefrom. Targetspecific magnetic resonance energies in the PicoTesla range are now calculable with a new particle-wave equation, $\mathrm{mc}^{2}=\mathrm{BvLq}$, known as Jacobson Resonance. The slowing of our biological clocks through regulation of telomere length may be possible, utilizing extrinsically sourced non-ionizing, non-significant risk magnetic fields [1].

Human telomeres are repetitive, non-coding DNA (TTAGGG) structures at the ends of chromosomes bound by a series of single and double-strand DNA - binding proteins. Telomeres shorten with each cell division, due to incomplete lagging strand synthesis. There are many published correlative studies demonstrating a connection between telomere length and aging, with evidence of an inherited component [2]. It has been hypothesized that when conformational states of protein and/or DNA are altered, this information is transmitted to the rest of the DNA through non-linear lossless vibration waves, or solitons; based in biological piezoelectricity. While end-processing events are less well understood, as is oxidative damage, we may presuppose that quantum state entropic changes are of relevance. Interatomic communications via electromagnetic forces are at the root of all signal transductive coupling mechanisms. When the binding protein for telomeres undergoes conformational changes in concert with incomplete lagging strand DNA synthesis (mechanical error), electromagnetic signals are sent to the rest of the DNA, and may inhibit normal genetic information transfer mechanisms. It is therefore projected that telomeres and/or binding protein may serve as targets for magnetic resonance energies, to maintain structural and functional integrity through photon-phonon transductions. It may also be possible to target telomerase (or components thereof) with subsequent inhibition and/or up-regulation as the result. Inhibition or up-regulation of telomerase may be provided by inducing enhanced coherent vibrational states via phonons. However, the resultant may be dependent upon the electrophysiological quantum environment of affected cells. Affectation of telomeres or telomerase then may be dependent upon changes already in occurrence, such as chromatin instability, DNA damage, and other stress signals such as overexpression of oncogenes. We note that various biological structures, e.g. keratin, collagen, alpha and beta sheaths of proteins, genes...etc. have been conceived to be piezoelectric. Whereas, these structures may form an uninterrupted reticulum that may act as quasi-crystalline piezoelectric networks, capable of converting electromagnetic oscillations to mechanical vibrations or vice-versa. Indeed, a diversity of studies has indicated that telomeric magnetic domains may prove susceptible to magnetic resonance energies in the physiologic Pico Tesla range [1-8].

It has also been demonstrated that Pico Tesla range magnetic 
fields can modulate autonomic nervous system tonicity by affecting vagal and sympathetic innervation [1,9,10,56,88]. Environmental stressors stimulate HPA-axis production of cortisol, whose primary action is to stimulate gluconeogenesis for production of heat energy to areas that need it most, e.g. the brain and major muscles. The heat energy exigencies demanded by environmental stressors may imbue a progressive slow burn in small volumes of tissues over time; thus increasing quantum entropic states, desiccation of microstructures, and microscopic scarring. In addition, excessive heat energies will produce disruption of cooperative coherent interatomic communications. Pico Tesla range magnetic fields have been shown to renormalize intrinsic electromagnetic profiles of tissues, and to restore desired vagal tonicity, to diminish heat energy exigencies associated with enhanced sympathetic innervation.

It is further hypothesized that target specific restoration of physiologic electromagnetic profiles may induce particle jumps, such that transposed immunogenic quantum domains may be renormalized; thus restoring biological order and atomistic/molecular coherent communications and cooperativity $[1,11]$.

\section{Fine tuning the jacobson theory of cancer and aging}

Enrico Fermi turned his attention to experimental nuclear physics in 1934, inspired by Chadwick's discovery of the neutron and the Joliot-Curies' production of artificial radioactivity. He reasoned that the neutron, being uncharged, could penetrate the nucleus easily and could trigger nuclear reactions. In this vein he discovered new radioactive isotopes. Although Fermi thought that he produced transuranic elements with uranium, Noddack proposed that Fermi had actually split the nucleus in two much lighter elements. The average binding energy of a nucleon in the uranium nucleus is $7.59 \mathrm{MeV}$ while the neutrons used in Fermi's experiments had energies of less than 1 $\mathrm{eV}$. How could a single low-energy neutron possibly split the uranium nucleus? Hahn and Meitner repeated Fermi's experiments, but it was not until Strassman urged Hahn to repeat the experiments that it was realized that the uranium nucleus had been split into isotopes of barium and the rare earth elements. It was Meitner and Frisch who worked out a rough picture of how the uranium nucleus, upon absorbing a neutron, distorts and splits into two roughly equal parts. Indeed, they calculated that the fission of uranium should be a highly exoergic reaction in which about $200 \mathrm{MeV}$ of energy are released, about ten times more energy than that of any previously known nuclear reaction [98]. Indeed, it was Lise Meitner who realized Einstein's equation, $\mathrm{E}=\mathrm{mc}^{2}$, explained the energy source.

The Bohr-Wheeler theory of nuclear fission says that the nucleus behaves like an incompressible drop of liquid. Nucleons are packed closely together, held by strong forces which cause the nucleus to assume a roughly spherical shape, just as a drop of liquid assumes a spherical shape to minimize surface tension. The nuclear forces play the role of surface tension and bring the nucleus into minimum-energy spherical shape. Now, before we continue our story we should look at the changing shape of a cell dividing - that is the deformation of a roughly spherical shape to an ellipsoidal shape. After prophase and the migration of centrioles, the cell moves to metaphase, wherein chromatids appear after the process of condensation. The double nature of mitotic chromosomes becomes evident at metaphase, by which stage-two chromatids can be distinguished. Thus the cell's chromosomes all become arranged in the same transverse plane. This plane crosses the middle of the cell at right angles to its spindle axis in the equivalent position to the world's equator. This arrangement is assumed as a result of the development of a second set of microtubules. Until anaphase, the two chromatids of a mitotic chromosome are joined at the centromere, where a kinetochore is present on each chromatid. The centromere region appears in the LM as a constriction. Now, returning to our story of fission, we note that when the nucleus absorbs a neutron, it is deformed into an ellipsoidal shape much as the mitotic cell. Yet the nuclear surface tension tends to restore the nucleus back to a spherical shape, and oscillations set in. If these oscillations are large enough, a "neck" will form, and the nucleus will assume a dumbbell shape. Returning to our dividing cell, we see in telophase the constriction of the middle of the cell becomes a cleavage furrow that deepens and eventually pinches the cell into two. Of course in nuclear fission, a separation of charge is created, and the positive charges in the two halves of the dumbbell repel each other. If the shape of the nucleus is too deformed, the nuclear force will not be strong enough to overcome this repulsion (keeping in mind that the nuclear force has a much shorter range than the electrostatic force), and the nucleus will split into two roughly equal parts. With the Bohr-Wheeler theory it was possible to estimate the activation energy needed to deform a nucleus so that is splits. Noting that nuclei of intermediate size have the highest bonding energies per nucleon and therefore are more stable than lighter and heavier nuclei, we must question whether the fission process may occur in biological systems enhanced by predisposition of proto-oncogenes and biologically asymmetrical structures and invasion of foreign bodies; e.g., viruses and chemicals, into the genome which regulates cellular function in an orderly fashion for life. If a heavy nucleus is split into two smaller ones, the greater binding energy of the latter means that energy will be liberated. This may be analogous to the growth process in biological systems wherein as cells divide, more energy is liberated. Certainly youth maintains more energy than is expressed by mature organisms. Since the products of the fission include several neutrons as well as two daughter nuclei, a chain reaction can be established in an assembly of a suitable fissionable isotope. If it is uncontrolled, as in cancer, the result is an atomic bomb. If it is controlled so that the rate at which fission events occur is constant, the result is a nuclear reactor that can serve as an energy source for generating electricity; the basis of life and the explanatory mechanism of B. Nordenstrom's concept of biologically "closed" electric circuits, which identifies biosystems as intrinsic, closed, self-perpetuating mechanical agglomerations of matter, in a very real sense [98-101].

The activation energy for fission can generally be supplied in two ways: 1) by energy released in the neutron reaction; and 2) by the kinetic energy brought in by the captured neutron. However, it is known that there is also a smaller probability that a nucleus will accidentally absorb sufficient energy through collisions or other random processes and spontaneously split without being hit by a neutron. Although the likelihood of spontaneous fission in heavy nuclei is generally rather small, it nevertheless provides an explanation for spontaneous fission in a cell leading to a chain reaction - cancer $[98,102-104]$.

In the cyclotron, when a charged particle is introduced into the center, it is accelerated by an electric field across a gap and deflected onto a circular path by a uniform magnetic field. With each crossing of the gap, the particle gains energy and the circles grow wider. In looking at the orientation of structures in a cell during prophase, we see the divergence of centrioles (piezoelectric structures) and the possible production of an increasingly greater regulation of a changing electric field throughout the cell which is a polarized structure itself. The appearance of threadlike chromosomes, disappearance of the nucleolus, disintegration of the nuclear envelope and forming 
microtubules indicates an increasing acceleration of particles due to influx of energy $(\Delta \mathrm{E})$ and an increasing number of collisions. The alignment of chromosomes with their centromeres in metaphase along with formation of chromosomal microtubules, and continuous microtubular structures, and creation of an elliptical shape within the deforming cell, may indicate that a cyclotron resonance circuit is acting to promote increased speed of particles across the space between chromatids. In a controlled state, the ever-widening circular paths and particle accelerations may be limited (normal mitosis) by carbon atoms, as they can serve to slow down the fission process. Biological controls in normal mitosis may diminish particle interactions and random collisions with control structures, either absorbing neutrons and/or other wave particles; e.g., gamma rays. Physiologic static magnetic fields on the order of $5 \mathrm{pT}$ have been measured to be associated with delta brain waves at MIT. In a cyclotron a particle gains energy from changing electric fields and this feature may be adjusted in a biosystem by structures external to the cell; i.e., extracellular matrix which is also piezoelectric. The steady magnetic field in a cyclotron and biosystem serves merely to move particles in circular paths without increasing velocity. Thus the effect of cyclotron resonance in a biological system would preserve compactness of the system such that the magnetic bending allows particles to be accelerated by the same electric field over and over again confining the paths of particles to relatively small areas, thus accounting for circadian rhythms in biosystems as well. In the normally dividing cell it is hypothesized that the regulation of the fission process is produced by the extracellular matrix which envelops the dividing cell as it splits into two daughter cells, and in a very real sense the filamentogenic structures of extracellular matrix act as control rods which absorb enough of the randomly moving particles to prevent a chain reaction. The frequency of the oscillating phenomenon related to hydrogen bonds is not the same whatever the situation. It does appear to remain from near IR $\left(10^{14} \mathrm{~Hz}\right)$ to far IR neighboring mm MW $\left(10^{12}-10^{11} \mathrm{~Hz}\right)$. The oscillation frequency of $\mathrm{HB}$ in DNA is in the lower range and about $10^{11} \mathrm{~Hz}$. These hydrogen bonds can be considered to be EM radiation emission centers. The internal regions of proteins favor the creation of hydrogen bond networks producing in the extracellular matrix and cytoskeletal system (including actin and ATP molecules) proton semi conduction systems. The HB networks act as proton conductors and are responsible for photon-conformational interactions able to do biophysical work. The production of solitons is also a regulative mechanism for absorption of wave particles, which can stop the fission process in normal mitosis. [13,14,19,36].

Hydrogen bonds in cells act as piezoelectric centers of energy transduction with biophysical characteristics, which probably depend upon their length, explained by Jacobson Resonance. According to Volkenstein, $[13,105]$ the passage of a proton along channels of hydrogen bonds ( $\alpha$-helices and $\beta$-sheets) can alter the frequency of the EM radiation emitted by the hydrogen bonds they contain, by which protonic conformational interactions may signal the change in the protein conformation at a distance and in real time, as well as performing the mechanical work (such as adaptation between enzyme and substratum). In ordered biosystems, because of the very large number of $\mathrm{HB}$ in the cell, EM emission from a complex harp orchestra takes place uniformly and in all directions. In cancer, uniformity and unidirectionality is lost and the system breaks in chaotic, heterogeneous models which use more energy $(\Delta E)$ from the normal structures taxing their abilities to continue in performance. When we consider the tertiary structure of proteins and the various domains, $\beta$-sheets, $\alpha$-helices and relative $\mathrm{HB}$ chains that may have different positions within the macromolecule, then one may see that within the physiologic system all the intramolecular hydrogen harps are a source of EM radiation at a set frequency and intensity level. Clearly, uncontrolled fissionary processes will perturb normal structures to render them unable to cope with an ever-increasing number of particle accelerations and collisions producing polyploidy. The increasing level of configurational energy produces the increasing level of disorder of biostructures. In an ordered system, an expression of photomechanical ponderomotive forces generated by coherent electric forces propagates as filamented beams in a non-linear medium [13,19,51,96,99,106-109].

The number of asymmetries is projected to start the fission process. The fission process increases the heat and radiation - especially gamma rays, higher in energy and frequency than $\mathrm{x}$-rays. The gamma rays may promulgate the fusion process that represents the cancerous process [104]. How much $\mathrm{x}$-ray and gamma ray comes out of a biosystem may not be important. It is the differential between what is emitted by pathologic versus non-pathologic tissue and should be explored.

\section{Preliminary analysis and study of cancer}

\section{Our hypothesis with respect to cancer is the following:}

Firstly, there is predisposition of proto-oncogenes genetically transmitted. Then there is asymmetry produced by invading agents such as carcinogens, chemicals trauma, microorganisms and EM radiation (ambient). There is then mitosis, resulting from growth which is ordered by balancing symmetries of forces, or growth not in development but in repair which occurs normally in life. And, then there is mitosis specifically for production of energy as a result of stimulus that may be considered atypical. Fission produces energy needed by the body to function. Yet, if the asymmetrical force and/or forces continue, extra mitotic division will result - the ongoing fissionary process. If this occurs too long, then the ultimate quantum characters that relate life (EM field to gravitational field) must be emitted from biostructures as compensatory mechanisms. X-rays and gamma rays are emitted but not in large quantities (relative to radioactive substance which have heavy nuclei like uranium.) Small quantities of energy are emitted in discrete forms from small volumes of space containing high-velocity particles which are necessary for fission/fusion. The plasma or ionic masses are ignited in ongoing cancer and there is a thermonuclear fusion explosion. The body tries to restore order with its own energy supplies - thus the dramatic weight reduction of terminal cancer patients. Hypothetically, the way to control thermonuclear fission/ fusion is to externally improve reordering electromagnetic fields.

Preliminary studies at Mississippi State University executed by Coyne et al. [101], screened a number of PicoTesla range magnetic field schedules. Calculations using the Jacobson Resonance equation were based upon molecules thought to be associated with human mammary carcinoma cell populations (HTB-126 and MCF-7) in multi well tissue culture plates. Two schedules were found to compromise the viability and/or proliferation rate of HTB-126/MCF-7 cell types relative to untreated negative reference controls. Over the course of replicate studies $(n=7)$ these PicoTesla schedules were observed to consistently inhibit the viability and/or proliferation rate between $31 \%$ to $35 \%$ compared to untreated negative reference controls. Additionally, Coyne identified membrane-associated complexes that were expressed at elevated or decreased levels in MCF-7 populations. Several mRNA sequences were detected $(n=3)$ that were expressed at higher levels $(n=1)$ or uniquely expressed $(n=2)$ in MCF-7 populations. Interestingly, these cells were exposed to magnetic resonance energies for thirty minutes each time in five treatment sessions, compared to exposure times of fifty-six minutes, twice weekly, for 8.5 weeks as 
was executed in nerve regeneration studies at Fairleigh Dickinson University for the in-vivo study. It is the experience of the authors that outcome measures are generally directly related to exposure time- in addition to the conditions of resonance determined by accuracy of flux densities and frequencies.

Nevertheless, collective interpretation of experimental findings revealed an ability of a multi-frequency, multi-amplitude PicoTesla range magnetic schedule to induce alterations in viability/proliferation rate and expression profiles of: (1) cytosol-soluble and membrane associated protein fractions; and (2) genetic transcription of mRNA sequences compared to negative (non-exposed) reference controls. In this context, these alterations appear to be of a different pattern when experimental samples were immediately processed following MCF-7 exposure to the fifth and final schedule. In contrast, different and slightly more subtle differences were appreciated when an intentional delay of several hours was implemented between the final exposure and sample preparation. Appreciation of this observation implies that maximum alterations in protein expression and mRNA transcription may occur during or shortly after periods of exposure. In addition, there was also a relative difference in the biological affect excited by individual flux density/frequency techniques contained within the "Master" multi-frequency PicoTesla schedule. Ultimately, these laboratory findings may serve as an experimental foundation for future research investigations devoted to delineating (1) time frames that PicoTesla magnetic fields exert a biological affect, (2) duration of PicoTesla magnetic field induced molecular/genetic alterations, (3) identity of PicoTesla magnetic fields that selectively exert specific biological affects in living systems, and (4) identify molecular/ genetic "targets" that PicoTesla magnetic fields interact with in a manner that creates a biological affect.

\section{What keeps cancer cells alive?}

Interestingly, cause and effect relationships in cancer are difficult to prove e.g. whether shortened telomeres initiate or prevent cancer. Correlative evidence might appear to show that telomere uncapping and resultant DNA damage signals may suppress cancer cell cycle checkpoints, because the damage cannot be repaired in the absence of telomerase. However, when alterations in specific cell cycle regulatory pathways, e.g. inactivation of $\mathrm{p} 53$, cells can continue to divide still having critically shortened telomeres, while expressing DNA damage pathways. This is a bypass of senescence (M1) with cells continuing to divide. When many telomeres are shortened to the extent that end end chromosome fusions occur, this produces chromosome - breakage - fusion - bridge cycles known as M2 or crisis. Crisis stage M2 leads to extensive chromosomal alterations, the hallmark of cancer. It is hypothesized that transpositional states based in quantum entropic conditions lead to transitions from altered cell cycle checkpoints encouraging cells to grow to meet increased energy exigencies. Terminally shortened telomeres may not prevent cell divisions in a rarity of human cells that can bypass this stage. This type of rare cell manifests the hallmarks of telomere stability with reactivation of telomerase. The fundamental mechanisms underpinning a bypass of senescence (M1) and continuance to the crisis point (M2) may be based in quantum phenomena which ultimately determine such biochemical changes. Actively dividing cancer cells could then survive and continue to replicate. Further hypothesized is the idea that an electromagnetic physiologic immune response provides the encouragement for certain cells to divide and grow while avoiding crisis stages, in spite of having terminally shortened telomeres. This explanation is proposed for contrasting normal mitosis versus neoplasia.
It is further hypothesized that MRT may stimulate up regulation of telomerase when telomere length diminishes too greatly in specific tissues, while functionality has decreased. Telomerase is projected to be a defensive mechanism of the body, because telomerase increases telomere length. In cancer, when telomeres become too shortened, telomerase is up-regulated to increase telomere length, but in crisis stages, M1 -M2, quantum state entropy is too great for the defensive mechanism to overcome the anarchy of cells. Thus, telomerase produces increased lengths of telomeres in actively dividing cancer cells, therein keeping the cancer cells dividing and surviving; and as actively mitotic cells increase energies from fissions/ fusions of subatomic particles, the cancer cells increase energy, whereas the chain reaction proceeds. In simple terms, the cancer cells have superseded the normal cells while using the natural defense mechanisms of normal cells to survive and multiply. The degree of chaos/quantum entropy from fissions/fusions overcomes the natural defense mechanisms based in telomerase production.

\section{Considerations of specific target mass magnetic resonance energies for research}

We may query whether magnetic resonance energies can maintain the structural and functional integrity of telomeres and telomerase. Reiterating, telomeres are essentially biological clocks that decrease in length with age, and once a critically shortened telomere length is attained, cellular senescence may be activated. When a sufficient number of cells undergo senescence in a tissue, a decline or loss of function will occur that could contribute to aging or cancer. Telomeres shorten due to incomplete lagging strand synthesis, and when conformational states of proteins and/or DNA are altered, this information may be shared with the rest of the DNA via piezoelectric messaging. Single or double strand DNA- binding protein and/or DNA may be targetable. Let us first consider the primary structure of a telomere as our target mass. Consider the telomere unit (TTAGGG) Whereas, $\mathrm{T}=258.164$; $\mathrm{A}=267.176 ; \mathrm{G}=299.176$; and TTAGGG=1,681.032 daltons. Thus, the molecular mass of the prime telomere unit is $1,681.032 \mathrm{Da}$. Considering $\mathrm{Mc}^{2}=\mathrm{BvLq}$ :

$(1,681.032 \mathrm{Da})\left(1.67 \times 10^{-24} \mathrm{gm}\right)\left(9 \times 10^{20} \mathrm{~cm}^{2} \mathrm{sec}^{-2}\right)=\mathrm{mc}^{2}$

$=(\mathrm{B})\left(3 \times 10^{6} \mathrm{~cm} \mathrm{sec}^{-1}\right)(177 \mathrm{~cm})(1 \mathrm{ab}-$ coul $)=\mathrm{BvLq}$

$\mathrm{B}=4.7581 \times 10^{-9}$ Gauss

Now, $\mathrm{f}=10 \mathrm{qB} / 2 \pi \mathrm{m}$, and we desire:

$$
\mathrm{f}=\frac{(10)\left(1.6 \times 10^{-20} a b-c o u l\right)\left(4.7581 \times 10^{-9} \text { Gauss }\right)}{(6.2832)\left(9.11 \times 10^{-28} \mathrm{gm}\right)}
$$

And, $\mathrm{f}=0.133 \mathrm{~Hz}$

Therefore, the essential signal parameters for the telomere prime unit are:

\section{$4.7581 \times 10^{-9}$ Gauss @ $0.133 \mathrm{~Hz}$}

We must consider the forgoing as the prime set of signals, because the domain walls of the essential target represent magnetic interfaces of energy domains. Integral multiples of the prime may then be utilized within the context of a master protocol to specifically influence tissues of concern. For example, the protocol used successfully to diminish the viability of human mammary cancer cells included integral multiples of the prime telomere signal starting from about 15 picoTesla down to about 3 picoTesla. Included within the context of this master protocol 
were interleukins and interferons as well as TNF. Most interesting is the fact that integral multiples of prime telomere signals not only include signal sets that target cytokines but also specific tissues such as tendon and brain. Considering the flux density of PTEMF's used to treat Parkinson's disease, we note that the $\mathrm{B}$ value was about $7.5 \mathrm{pT}$, whereas the $B$ value utilized to affect cardiac rate and rhythm was about $3.4 \mathrm{pT}$ in experimental studies at the University of Oklahoma Health Sciences Center. We also note that telomerase associated proteins such as NOP10, NHP2, GARI, and Dyskerin reveal molecular masses analogous to telomere prime integral multiples, cytokines and tissues specific magnetic resonance energies including the brain and the heart.

Our essential hypothesis is that even though telomeres are non-coding DNA sequences, tissue specific PTEMF's empirically determined may be affecting telomeres of specific lengths (and specific molecular masses) to therein provide for up-regulation of telomerase; with distinct relationships to other critical molecules (in the absence of neoplasia). Table 1 shows integral multiples of the prime telomere unit (TTAGGG), associated flux densities and frequencies of magnetic

Table 1. Multiples of the telomere prime $(\mathrm{L}=177 \mathrm{~cm})$.

\begin{tabular}{|c|c|c|c|}
\hline & $\beta(\mu G)$ & f (Hz) & mass (kDa) \\
\hline 1 & 0.004758100 & 0.133174371 & 1.681032000 \\
\hline 2 & 0.009516200 & 0.266348742 & 3.362064000 \\
\hline 3 & 0.014274300 & 0.399523113 & 5.043096000 \\
\hline 4 & 0.019032400 & 0.532697484 & 6.724128000 \\
\hline 5 & 0.023790500 & 0.665871855 & 8.405160000 \\
\hline 6 & 0.028548600 & 0.799046226 & 10.086192000 \\
\hline 7 & 0.033306700 & 0.932220597 & 11.767224000 \\
\hline 8 & 0.038064800 & 1.065394968 & 13.448256000 \\
\hline 9 & 0.042822900 & 1.198569339 & 15.129288000 \\
\hline 10 & 0.047581000 & 1.331743710 & 16.810320000 \\
\hline 11 & 0.052339100 & 1.464918081 & 18.491352000 \\
\hline 12 & 0.057097200 & 1.598092452 & 20.172384000 \\
\hline 13 & 0.061855300 & 1.731266823 & 21.853416000 \\
\hline 14 & 0.066613400 & 1.864441194 & 23.534448000 \\
\hline 15 & 0.071371500 & 1.997615565 & 25.215480000 \\
\hline 16 & 0.076129600 & 2.130789936 & 26.896512000 \\
\hline 17 & 0.080887700 & 2.263964307 & 28.577544000 \\
\hline 18 & 0.085645800 & 2.397138678 & 30.258576000 \\
\hline 19 & 0.090403900 & 2.530313049 & 31.939608000 \\
\hline 20 & 0.095162000 & 2.663487420 & 33.620640000 \\
\hline 21 & 0.099920100 & 2.796661791 & 35.301672000 \\
\hline 22 & 0.104678200 & 2.929836162 & 36.982704000 \\
\hline 23 & 0.109436300 & 3.063010533 & 38.663736000 \\
\hline 24 & 0.114194400 & 3.196184904 & 40.344768000 \\
\hline 25 & 0.118952500 & 3.329359275 & 42.025800000 \\
\hline 26 & 0.123710600 & 3.462533646 & 43.706832000 \\
\hline 27 & 0.128468700 & 3.595708017 & 45.387864000 \\
\hline 28 & 0.133226800 & 3.728882388 & 47.068896000 \\
\hline 29 & 0.137984900 & 3.862056759 & 48.749928000 \\
\hline 30 & 0.142743000 & 3.995231130 & 50.430960000 \\
\hline 31 & 0.147501100 & 4.128405501 & 52.111992000 \\
\hline 32 & 0.152259200 & 4.261579872 & 53.793024000 \\
\hline
\end{tabular}

resonance energies, and the molecular mass analogs.

\section{A different perspective on the relation of quantum phenomena, the injury potential and cancer}

Bjorn Nordenstrom [29,30] found a cosmos of electrical activity in the human body providing the foundation of healing, and critical to health as the flow of blood. Nordenstrom's vascular interstitial closed electric circuit (VICC) is activated by normal metabolism with respect to degrading processes such as spontaneous necrosis and hemorrhage. The VICC leads to local electrochemical polarization of a lesion relative to surrounding non-injured tissue. Ions, cells, and water are transported electrically into the developing corona complex associated with pulmonary cancer. The walls of arteries and veins function as insulators around the electrically conducting medium of blood, whereas blood vessels connect injured and non-injured tissues at the capillary level, and interstitial fluid completes the biologically closed electric circuit (BCEC) Thus, there is a slowly fluctuating, attenuating electrochemical potential inducing ebb and flow of time-dependent anionic and cationic transports, based in the corona structures of pulmonary cancers seen on radiographic analysis. Nordenstrom termed this manifestation the corona structure because of its resemblance to the sun's corona. Corona structures of the lungs may be recognized in twenty to thirty percent of patients whose tumors are peripheral to the hila and larger than 2.5 centimeters. The corona structure contains "A" and "B" zones. The A zone is characterized radiographically by radio lucency around an electrically polarizing focal lesion. Peripheral to the $\mathrm{A}$ zone, a $\mathrm{B}$ zone is seen as a radiopaque region. The $\mathrm{A}$ and $\mathrm{B}$ zones are basically the result of a electro-osmotic flow of water from a lesion during its electropositive phase. At the interface between A and B zones, small arches sometimes form and arcade. This configuration develops when the polarizing lesion has small protrusions at its surface. As a result of electrical edge enhancement and interface interactions, various elements of the interstitial tissue are transformed into radiating fibrous structures. They grow out at right angles to the surface of the lesion and serve as supporting columns for the arches. When necrotic material from a tumor is evacuated through a bronchus, collapse of the tumor follows and will displace radiating structures already produced. They then present as regular elongated opacities termed lamellae. Experimental production of A and B zones, the radiating structures, arches and arcades have been presented [29,30] (Figures 2 and 3). Circumferential structures are said to be related to perifocal edema due to dystrophic changes in the lung parenchyma by microthrombosis of vessels around the lesions. Perifocal edema is related to electro osmotic inflow of water to the mass during its electronegative phase, and contributes to a circular displacement of structures. Additionally, local dehydration by electro osmosis may lead to shrinkage of hydrophilic fibro elastic tissue, producing a structural change, e.g. skin thickening also related to breast cancers.

The discovery of the corona structures enable a correlation of the fractionally quantized Hall effect (FQHE) to biosystems. The FQHE indicates a two-dimensional quantum liquid of electrons rendered incompressible by the density quantization of its ground states, whose excitations are quasi-particles of fractional electric charges. The quantized Hall effect is a remarkable macroscopic quantum phenomenon occurring in semiconductors at experimentally produced low temperatures in two-dimensional electron systems, subjected to high magnetic fields.

It was Von Klitzing who first noticed conductivity plateaus while reviewing semiconductor carrier densities and the elementary theory of 


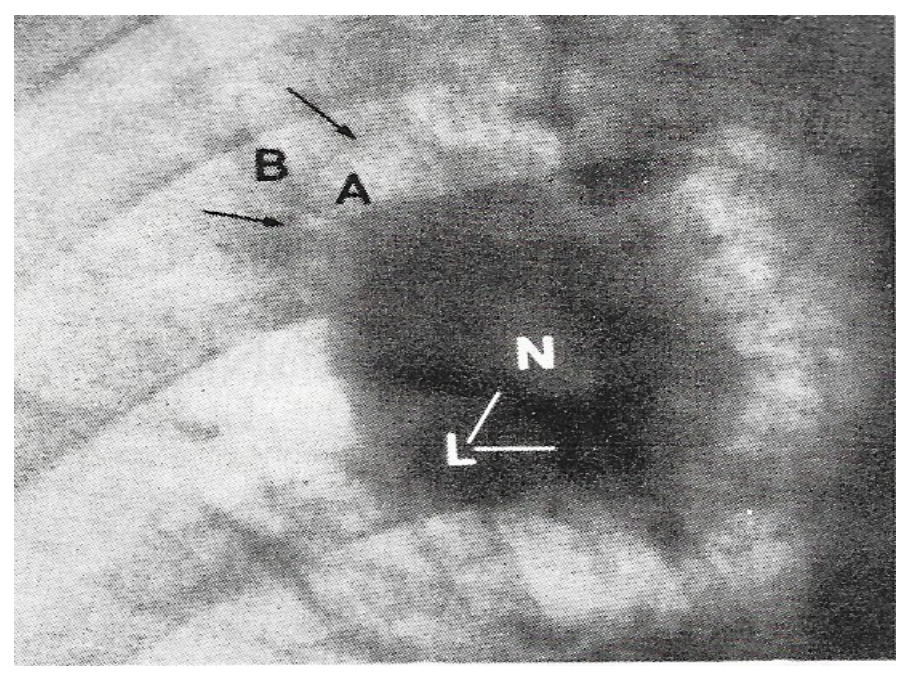

Figure 2. This shows a well-differentiated adenocarcinoma of lung (frontal radiograph). A 1-2 cm radiolucent, " $A$ " zone surrounding the tumor. A " $B$ " zone is seen peripheral to the " $\mathrm{A}$ " zone, and the "B" zone is smaller and somewhat more radiopaque. The central area of the cancer presents as necrotic and ill defined. The central radiolucency of which is labeled $(\mathrm{N})$. Note radiating structures from the tumorous surface, and a y-shaped band called lamellae (L) overlie the mass. At the distinct interface between the zones, note a $2 \mathrm{~cm}$ long arcade (see arrows). Close inspection suggests the presence of small arches formed this structure, each of which is about $1-2 \mathrm{~mm}$. (Nordenstrom B. BCEC. Nordic Medical Pub 1983:15)

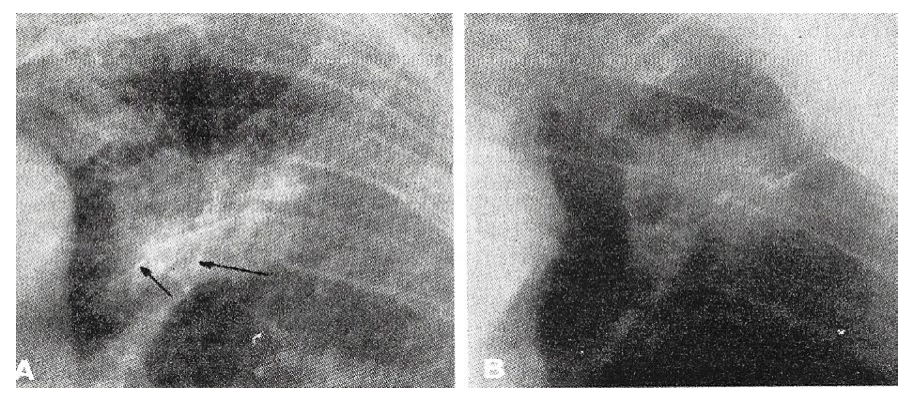

Figure 3. This shows a moderately well differentiated squamous cell carcinoma, with lamellae. These linear opacities appear to cross one another over the tumor in posteroanterior projection (A arrows). In slight left posterior oblique projection (B), lamellae are projected at the surface of tumor. (Reprinted from AJR, Sept. 1985; 145:157; Figure 17).

Landau level filling in the inversion layer of a silicon Mosfet transistor. Von Klitzing exploited the Integral Quantum Hall Effect to achieve a high precision measurement of $\mathrm{e}^{2} / \mathrm{h}$, a fundamental constant of nature, hence a better determination of the fine structure constant. To the nearest ohm, h/ $\mathrm{e}^{2}$ has a value of $25,813 \mathrm{ohms}$. In this theory, $\mathrm{e}^{2} / \mathrm{h}$ is the fundamental unit of 2 dimensional conductivity.

Furthermore, the fractionally quantized Hall Effect discovered at Bell Labs shows fractions of $\mathrm{e}^{2} / \mathrm{h}$, which one can only explain in terms of electrons in a chorus. When the chorus line is disturbed, and a kink develops in the line, e.g. due to a physiological adjustment in a biological system, a production of the shortening of the distance between electrons produces a structure that acts like a particle. A totality of adjustments in strings of electrons acting collectively creates the quesi- particle with precisely one third the charge of an electron. The electrons will execute cyclotron orbits about magnetic field lines according to $\mathrm{f}=\mathrm{qB} / 2 \pi \mathrm{m}$. The cyclotron accelerates a beam of energetic particles in spiral paths at right angles to a constant magnetic field from an AC electric field. This has been likened to the system of a unit cell $[29,30,45,107]$.
The critical point demonstrated by Nordenstrom, that there is a slowly fluctuating attenuating electrochemical potential inducing ebb and flow of time dependent anionic and cationic transports based in the corona structures, guides one to the picture of magnetic resonance in electrical systems. Inductance is analogous to inertia, capacitance is analogous to elasticity. One keeps in mind the rather straight relaxed radiating elastic structures described by Nordenstrom and the fact that very low temperatures were utilized to demonstrate the quantized Hall Effect as electronic collisions are most elastic at temperatures below 4 degrees Kelvin.

Indeed, the capacitive coupling of vessel walls drive the VICC, and the inductance of looping created by the cyclotron resonance effect may be a fundamental mechanism of quantized corpuscular and wave adjustment.

While a stretched spring stores mechanical energy, a capacitor stores electricity in its natural state. In its simplest form, a capacitor is made up of two parallel metal plates separated by an insulating material such as air or mica. In Nordenstrom's system, the capacitor is any substance capable of storing electrical charge based upon its relative resistivity to surrounding structure. For example, the average resistivity of human tissue, falls within the range of $80-120$ ohms per centimeter as a general statement. Nordenstrom points out that the resistivity of the walls of capillaries is 200-300 times greater than that of the blood plasma. This means that we can see a direct numerical correlation to $\mathrm{h} /$ $\mathrm{e}^{2}(25,813 \mathrm{ohms})$, based upon the resistivity of the insulator cables or capillary walls which comprise the capacitive factor of the VICC; the system which connects the lesion to the surrounding tissue electrically $[29,30,98,107,45]$.

Additionally, when we place a large number of electrons into a resonance circuit, as it is stimulated electrically, the electrons will surge through the system and accumulate on the capacitor on the opposite side of the system. After the electrons have completed the passage, the current stops, and the same thing may take place in the opposite direction. To understand the role played by the resistivity of the tissue, remember that inductance is really electrical inertia. The current would prefer to stop when the electrons are equally distributed between the two plates of the capacitor, but it cannot stop instantaneously any more than a car can stop the very instant the breaks are applied. Returning to a mechanical analogy, a weight suspended by a spring passes through its normal rest position many times as it bobs up and down. It doesn't stop at its rest position immediately because of its inertia, and it can come to rest only after many oscillations during which the energy of motions is slowly changed into heat by friction. The same process takes place in the resonance circuit in the human body as the current surges back and forth from insulator to insulator in the VICC. Electrical resistance in the capillaries in the VICC plays the part of friction in slowly changing the electrical energy into heat; and the resonance frequency of a circuit is determined primarily by its inductance and capacitance. More specifically, the inductance is based upon inertia of the point masses or quantum particles, the cells and ions...etc., as they traverse the VICC system. It is important to understand that because of the complexities and irregularities of human tissue, the human body may be considered a semi conductor. The resistance of human tissue ranges generally from about the resistance of germanium ( $55 \mathrm{ohm}$ per centimeter) to the resistance of silicon $\left(5.5 \times 10^{4} \mathrm{ohms}\right.$ per centimeter). However, it may be inferred from the distance from conduction bands, resistivity of condensed chromatin and chromosomes may approach a million ohms per centimeter of spatial line extension. Indeed, it appears probable that quasi-particles may well participate in the fissionary process, as 
entropic states provide kinks in chorus lines of electrons to promulgate creation of virtual particles, most especially if PicoTesla magnetic fields represent quantum gravitational states. Adjustment mechanisms may include solitons, a product of electrogravitational interactions that open worm holes to states of reality outside of ordinary matter, but included within the context of the total field.

Thus, photon-phonon transductions arguably must exist in all material bodies, especially because all ordinary matter is nothing else than condensed electromagnetic field. The description of fluctuating electro osmotic potentials within the VICC system conform to the law of conservation of mass and energy. Whereas, the flux seen in an oscillating steady state system, e.g. biological systems, immunologically adjusts order as entropy is increasing. This idea leads to an appreciation of the importance of streaming potentials, as well as cyclotron resonance (which is unitarily equivalent to magnetic resonance). The radius of the path described by any particle in vortical motion increases as the velocity increases, according to $\mathrm{v}=\mathrm{Her} / \mathrm{m}$. As the conductance of the tissue leads the current to approach the circumference of a corona structure about a tumor, and auxiliary electric fields deflect particles from the circular path as they leave through the interface. The electrical interface between the corona structures and the surrounding tissues determines the deflection path of radiating circumferential structures. The resistivity of the insulating cables (or the capillary walls) yields numerical correlations for the FQHE, the fractional Hall conductivity flux quantum $\mathrm{h} / 3 \mathrm{e}^{2}$. We see the quantization of Hall conductivity plateaus in fractions in the corona structures, as tissue resistivity approximates $7.8 \times 10^{4} \mathrm{ohms}$.

Furthermore, when a conductor carrying a current is exposed to a magnetic field, the Hall effect says an induced emf occurs in the conductor orthogonal to the magnetic field and the electric field. The Hall resistivity that is measured by solid state physicists is indeed equivalent to the resistance of human tissue. Whereas, a condition is created in the human organism as the electrostatic field $\mathrm{E}_{\mathrm{e}}$ and the non-electrostatic field $\mathrm{E}_{\mathrm{n}}$ are equivalent. This occurs when Landau level filling is completed, as adjustments take place. As the Fermi level is increased, leaving more Landau levels to be filled, fluctuations may continue as plateaus continue to be reached. The mechanism by which healing takes place involves fluctuating electrochemical and electrogravitational potentials permitting anionic and cationic, bosonic and quasi-particle transports to readjust homeostasis $[45,98,107]$.

Summarizing hypotheses: non-linear radiating structures emerging from the surface of a lesion give the lesion a hairy appearance, and are analogous to cyclotron resonance systems, wherein the electric field of the surrounding tissue expunges cells and ions from the circular path of the corona structure to leave in a looping path. The approximation of right angles is based upon the Hall effect, which describes a current that wants to traverse a path that is at right angles to the electric and the magnetic field. As a direct consequence of the foregoing, the arches and arcades that develop at the interface between (A) and (B) zones have been observed only in the presence of radiating structures which are generally one to two millimeters in diameter. As a group, the arches form a row, or arcade. The structures that emanate from the body of the lesion are intrinsically propagated by (\#1) the Lorenz force, orthogonal to the electric and magnetic fields internal to the lesion based upon the Hall effect and (\#2) contained therein magnetic domains moved in space by a physiologic AC magnetic field; to loop in a spiral as the magnetic field changes between the lesion and surrounding tissue, based upon the cyclotron resonance effect. The resistivity of tissues is altered to adjust chorus lines of electrons that determine production of quasi-particles. It is further hypothesized that there are numerous magnetic domains of varying electro-potentials that are independent energy systems- that must act coordinately to produce crystalline interfacing effects, e.g. resulting in formation of lamellae, having charged density differentials. Coordination of systems through space requires gravitational interactions based in solitons (affectations by gravitons). It is our purpose to draw attention to the underlying physical principles of quantum mechanics that may be involved in microscopic, mesoscopic and macroscopic phenomena, perhaps explaining radiographic manifestations exemplifying various physiological processes of human and biological systems. Greater understanding from a quantum point of view of biological phenomena in normal and diseased tissues will perhaps enable clinicians, experimentalists and theorists to determine greater numbers and better quality procedures to treat pathological conditions in the future. The quantized Hall effect is a remarkable macroscopic quantum phenomenon whose most striking manifestation is the appearance of conductivity plateaus at multiples of $\mathrm{e}^{2} / \mathrm{h}$ with an astonishing precision quite oblivious to the imperfections or geometric details of the semiconductor interfaces. It is the contention of this presentation that in accord with the Hall effect, when an exogenous nonionizing EM field is applied to the human body, a current carrying conductor, a small EMF is generated longitudinally across the body, and when the transverse Lorenz force is orthogonal to the magnetic and electric fields, i.e. the long access of the body is at right angles to the magnetic flux lines, an open circuit is created adjusting current density until $\mathrm{E}_{\mathrm{e}}=\mathrm{E}_{\mathrm{n}}$, or the changing magnetic field equals the steady magnetic field. Therein, a potential difference is generated between the domain walls of atomic crystal lattice structures producing a structural deformation of the lattices to bring about particle jumps e.g. gene jumps and atom jumps, thereby adjusting the spatial statistical orientation of organic molecules as Landau levels fill while Fermi energies rise.

While very low temperatures and strong magnetic fields were utilized to demonstrate the quantum Hall effect, there is no doubt that super conduction at very low temperatures occurs in biological systems, e.g. chills accompany fever. As well, extremely high temperatures in exceedingly small volumes of space account for biological fusions. A biological system is a universe of electro dynamical and gravitational interactions, the most complex of systems known. Temperature is a product of particle interactions (cold or hot) and spatial line extensions of any condition may be reflected in varying manner within biostructures. Interfaces of energy domains determine whether a volume of space is to be hot or cold. Furthermore, while strong magnetic fields exert brute force on systems, the same effects may be obtained through natural adjustments from weak magnetic profiles on quantum levels of structure and function. Controlled thermonuclear fusion reactions may be propagated through subtle, cajoling forces as opposed to brute force, overcoming repulsion of nuclei through quantum tunneling $[17,45,107,109,110]$.

\section{What are the limits of biological reversibility?}

\section{Nerve regeneration studies}

It is noted that the energy state and bioelectric potential of nerves may be modulated by electromagnetic fields (EMF's) $[12,81,83,86,88]$. For the following nerve regeneration studies, the field intensities, gradients and frequencies were calculated with the Jacobson Resonance equations; considering subcellular components vital for nerve function. Target molecules included nerve growth factors, dynein, kinesine, microtubule associated protein (MAP), neurofilaments, tubulin, 
cholinesterase, acetylcholine, and calmodulin. It was determined that the natural EMF profile for mice is in the micro gauss range $(1 \mu G$ $=100 \mathrm{pT}$ ). A sequence of extremely low-level EMF magnitudes with correspondent biological frequencies $(<300 \mathrm{~Hz})$ was employed.

The effect of these low-level, non-ionizing EMF's on the restoration of forelimb grip strength and radial nerve ultrastructure was studied in mice with induced motor neuropathy [12].

The Control Group $1(\mathrm{n}=10)$, was neither poisoned nor treated with EMF. Groups 2 and $3(n=20)$ were poisoned to induce motor neuropathy. Group $2(\mathrm{n}=10)$ after poisoning, was treated with EMF, while Group 3 was poisoned but not treated. Correlation of forelimb grip strength of all mice $(\mathrm{n}=30)$ at baseline was closely analogous (with no significant difference). Motor neuropathy was induced by administration of a neurotoxin (IDPN), in drinking water ad lib, for 9 $1 / 2$ weeks. Forelimb grip strength of mice, as measured by a force gauge meter, declined to $47 \%$ compared to the Control Group 1, a significant difference (Group 2, p $<0.004$; Group 3, $\mathrm{p}<0.00$ ). The normal age related increase in grip strength in the Control Group 1 was considered for the statistical analysis. The IDPN treated Group 3 (without EMF treatment) persisted to have a $56 \%$ decrease in grip strength; and radial nerve electron micrographs showed axonal demyelination, mitochondria in an orthodox state of conformation (inactive), and uneven dispersion of neurofilaments and microtubules. In contrast, IDPN treated Group 2 (with EMF exposure) exhibited axonal remyelination, condensed state of mitochondria (indicative of anabolic activity) and evenly dispersed neuro-filaments and microtubules, consistent with grip strength recovery (Figures 4-6).

EMF exposure was accomplished with the prototypical 18" Jacobson Resonator (Helmholtz configuration), built at the John C. Stennis Space Center by NASA engineers. Two mice at a time were held in two chambered ( 8 inch by 6 inch) Lucite perforated boxes. EMF's were applied twice weekly for $8 \frac{1}{2} 2$ weeks to Group 2 that resulted in $87 \%$

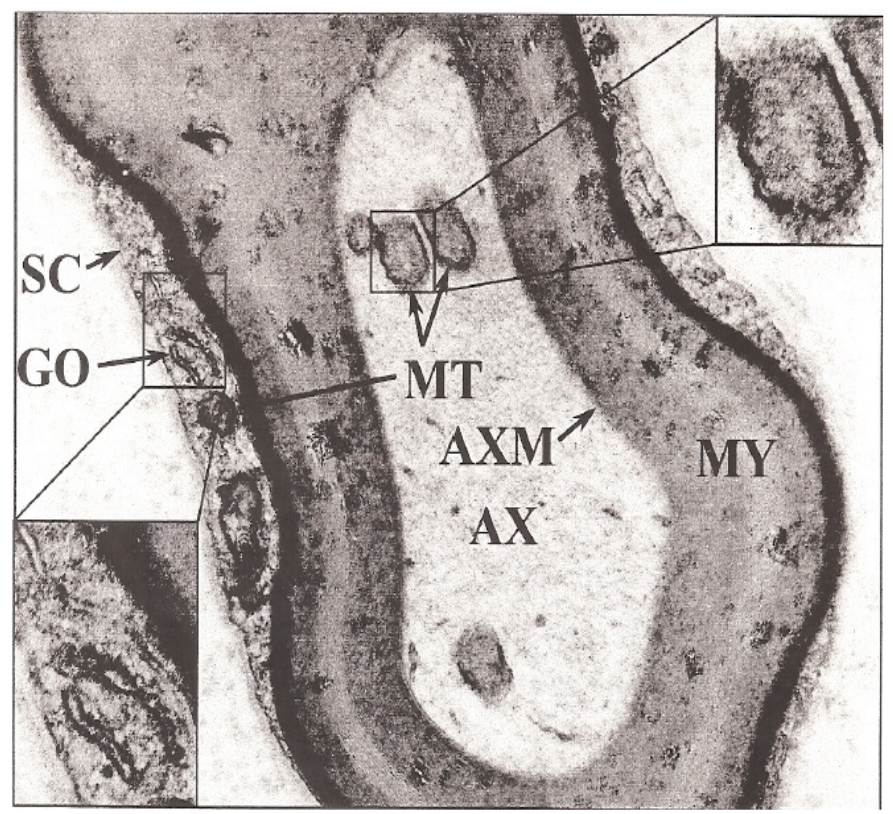

Figure 4. Electron micrograph (EM) of cross sections of radial nerve of mice from control Group 1, indicating Axon (AX), Axonal membrane (AXM), Golgi bodies (GO), Microtubule (MIC), Mitochondria (MT), Myelin sheath (MY), Neurofilament (NF), Schwann cells (SC), A. (Top) GO, MT, B. (Bottom Left) MT binary fission, C. (Bottom) NF. EM Magnification x 19,000. Scale Bar $=1 \mu \mathrm{m}$ Medical Hypotheses, 60(6): 821-839.
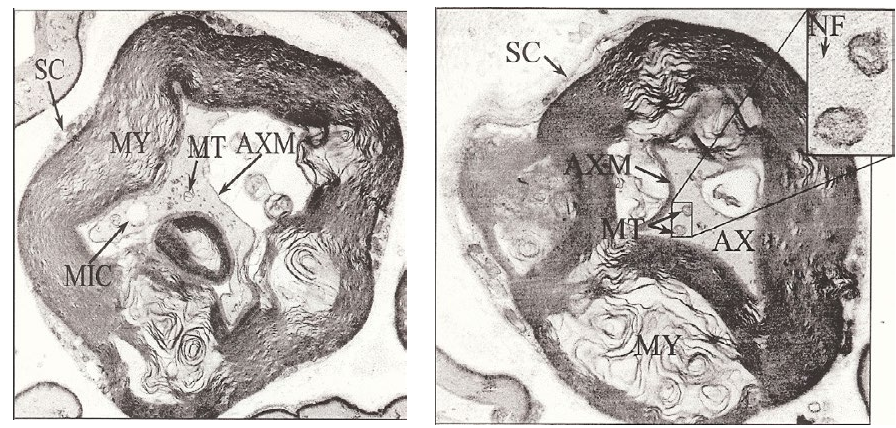

Figure 5. Electron micrograph (EM) of cross sections of radial nerve of mice from IDPN treated Group 3 unexposed to EMF indicating Axon (AX), Axonal membrane (AXM), Microtubule (MIC), Mitochondria (MT), Myelin sheath (MY), Neurofilament (NF), Schwann cells (SC). A. (Top) MY, MT, SC, AXM, MIC, B. (Bottom) MY, AXM, MT, NF. EM Magnification x 10,000. Scale Bar=1 $\mu \mathrm{m}$. Medical Hypotheses, 60(6): 821-839.

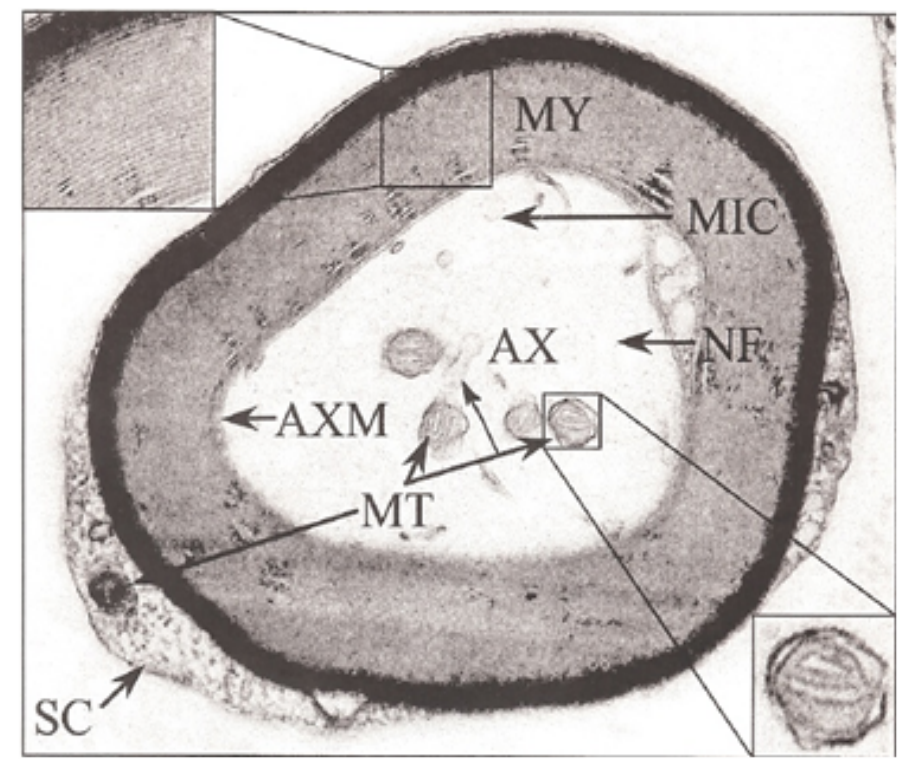

Figure 6. Electron micrographs (EM) of cross sections of radial nerve of mice from IDPN treated Group 2 exposed to EMF, indicating Axon (AX), Axonal membrane (AXM), Golgi bodies (GO), Microtubule (MIC), Mitochondria (MT), Myelin sheath (MY), Neurofilament (NF), Schwann cells (SC). A. (Top) MT, MY, AXM, NF, MIC, B. (Bottom Left) GO, MIC, C. (Bottom Right) GO, NF, MY, MT. EM Magnification A, B x 19,000, C x 4,800. Scale Bar $=1 \mu \mathrm{m}$. Medical Hypotheses, 60(6): 821-839.

recovery. $(\mathrm{p}<0.05)$ of grip strength that was sustained after termination of exposure at an $82 \%$ level until the $27^{\text {th }}$ week of observation. In the absence of EMF exposure, IDPN treated Group 3 had significantly low grip strength as compared to both EMF exposed Group $2(\mathrm{P}<0.01)$ and the control Group $1(\mathrm{p}<0.000)$ The neurotoxin effect persisted in Group 3 with $56 \%$ lower grip strength, as compared to Control Group 1. A consistent increase in grip strength, after termination of EMF exposure, was observed in Group 2 as it approached the level of the Control Group 1.

The gradual loss in forelimb grip test values in IDPN treated mice was indicative of a change in the nerve conduction in the forelimb. This was substantiated by an uneven distribution of axonal neuro-filaments, which determine growth of axonal diameters, and slow axonal transport for impulse conduction. The uneven dispersion of microtubules affected function in normal longitudinal growth and in fast axonal transport; and was a vital sign of nerve degeneration. The orthodox state of mitochondrial conformation indicates ADP deficiency. Reversal to a 
condensed state (EMF treated, Group 2) corresponds to an oxidative phosphorylation reaction and ATP synthesis, dependent on ADP, and proton permeability of mitochondria. Thus, Group 3, poisoned but not treated, showed reduced metabolic activity. Whereas, the condensed state of mitochondria in Group 2 (IDPN +EMF) indicated a metabolically active condition in axons and Schwann cells. In a previous study at Cornell, excised pieces of sciatic nerves of mice in-vitro culture medium maintained a normal myelin sheath structure and grew longer and wider during EMF exposure. This could be attributed to Schwann cell activity, a source of neurotropin for nerve growth. Schwann cells produce polypeptide nerve growth factors (NGF). Nerve injury induces an increased output of NGF from Schwann cells.

In the in-vivo study, two sources of NGF were available, one from CNS neurons and the other from Schwann cells. EMF exposure may have enhanced the action of Schwann cells in IDPN treated Group 2 mice. These Schwann cells indicated distinct Golgi bodies that are the source of NGF secretion, resulting in the remyelination of axons. Indeed, a link between non-ionizing EMF and renormalized Schwann cell function indicated that a non-neuronal control in the regeneration and growth of peripheral nerve fibers are definitely possible, i.e. the microscopic or quantum field regulation through inter-atomic and inter-molecular communications networks which were regulated by EMF. The renormalized, physiological state of mitochondria, as observed, indicated its normal membrane permeability and a recovery of ATP synthesis essential for nerve growth and repair. Other ATP dependent processes such as the organization of neurofilaments and microtubules for axonal slow and fast transport systems were also restored. The molecular signaling across an axonal membrane may be extensively modified by a low energy level of an applied EMF, and is attainable by cooperative amplification that can restore cellular function. A role of PTEMF (for humans) in recovery from nerve injury, spinal cord traumas, and peripheral neuropathies may be postulated on the basis of selectively modulating neurotropins and their receptors with PTEMF resonant energies [12].

\section{Conclusion}

Based upon the data gleaned thus far, it appears certain that non-ionizing radiation does indeed affect biological systems. It is the primary contention of this paper that stimulation of further research along the lines proposed is indicated.

\section{Acknowledgments}

I should like to thank my colleagues for their significant contributions to theory and experiment; William Yamanashi, Benjamin Scherlag, Brij Saxena, Bjorn Nordenstrom, Franco Bistolfi, and Ross Adey.

There is no conflict of interest.

\section{References}

1. Jacobson J, Scherlag B (2015) Aging and magnetism: Presenting a possible new holistic paradigm for ameliorating the aging process and the effects thereof, through externally applied physiologic PicoTesla magnetic fields. Med Hypoth 85: 276-286. [Crossref]

2. Gelmann EP, Sawyers CL, Rauscher III FJ (editors) (2014) Molecular Oncology: Causes of Cancer and Targets for Treatment. Cambridge University Press. UK; 2014:442-451.

3. Eichhorn GL (1979) Aging, genetics, and the environment: potential of errors introduced into genetic information transfer by metal ions. Mech Ageing Dev 9: 291301. [Crossref]

4. Hanahan D, Weinberg RA (2011) Hallmarks of cancer: the next generation. Cell 144: 646-674. [Crossref]
5. Slagboom PE, Droog S, Boomsma DI (1994) Genetic determination of telomere size in humans: a twin study of three age groups. Am J Hum Genet 55: 876-882. [Crossref]

6. von Zglinicki T (2002) Oxidative stress shortens telomeres. Trends Biochem Sci 27: 339-344. [Crossref]

7. Wu X, Amos CI, Zhu Y, Zhao H, Grossman BH, et al. (2003) Telomere dysfunction a potential cancer predisposition factor. J Natl Cancer Inst 95: 1211-1218. [Crossref]

8. Aviv A (2004) Telomeres and human aging: facts and fibs. Sci Aging Knowledge Environ 2004: pe43. [Crossref]

9. Wikswo JP, Barach JP, Freeman JA (1980) Magnetic field of a nerve impulse: first measurements. Science 208: 53-55. [Crossref]

10. Scherlag BJ, Yamanashi WS, Hou Y, Jacobson JI, Jackman WM, et al. (2004) Magnetism and cardiac arrhythmias. Cardiol Rev 12: 85-96. [Crossref]

11. Jacobson, JI, Scherlag B (2015) A dual resonance model for gerotargets and magnetic interaction energies. Telomere and Telomerase 2: 1-9.

12. Saxena A, Jacobson JI, Yamanashi WS, Scherlag BJ, Lamberth J, Saxena BB (2003) A hypothetical mathematical construct explaining the mechanism of biological amplification in an experimental model utilizing picoTesla (pT) electromagnetic fields. Med Hypoth 60 (6): 821-839. [Crossref]

13. Bistolfi F (1991) Biostructures and radiation order disorder. Edizioni Minerva Medica. Torino.

14. Bistolfi F (1991) A new approach to the interaction between ionizing radiation and bio structure: the dual compartment interaction model. Radiation Med 81(1): 99-108.

15. Wells D, Slusher H (1983) Physics for engineering and science. McGraw Hill, New York.

16. Kuchel PW, Ralston GB (1988) Oxidative Phosphorylation. Biochemistry. McGrawHill, Inc. New York.

17. Vergara W (1964) Electronics in everyday things. Harper and Row. NY 92.

18. Curie P (1887) Sur les phénomènes piézo-électriques. Bull Séances Soc Fr Phys $1887: 47-9$.

19. Jacobson JI (1991) A look at the possible mechanism and potential of magneto therapy. J Theor Biol 149: 97-119. [Crossref]

20. Marino A, Becker RO (1970) Piezoelectric effect and growth control in bone. Nature 228: 473-474. [Crossref]

21. Jacobson JI (1987) Oncogenes and magnetic resonance energies unified algebraically. Panminerva Med 29: 97-104. [Crossref]

22. Jacobson JI (1990) A testable theoretical model for magnetotherapy potentially applicative to such diverse concerns as oncogenic, CNS trophic factors, and viral disorders. Indian J Biochem Biophys 27(1): 58-62.

23. Marino AA, Cullen JM, Reichmanis M, Becker RO, Hart FX (1980) Sensitivity to change in electrical environment: a new bioelectric effect. Am J Physiol 239: R424427. [Crossref]

24. Bassett CA, Mitchell SN, Gaston SR (1982) Pulsing electromagnetic field treatment in ununited fractures and failed arthrodeses. JAMA 247: 623-628. [Crossref]

25. Jacobson JI (1996) Speculations on the influence of electromagnetism on genomic and associated structures. J Int Med Res 24: 1-11. [Crossref]

26. Jacobson JI (1992) "Exploring the potential of magneto-recrystallization of genes and associated structures with respect to nerve regeneration and cancer". Int J Neurosci 64: 153-165. [Crossref]

27. Jacobson JI (1994) Influence of electromagnetism on genes and associated structures Isr J Med Sci 30: 245-248. [Crossref]

28. Jacobson JI (1987) Oncogenes and magnetic resonance energies unified algebraically Panminerva Med 29: 97-104. [Crossref]

29. Nordenstrom B (1983) Biologically Closed Electric Circuits: clinical, experimental and theoretical evidence for an additional circulatory system. Nordic Med Pub 1983: 69-74, 112-150, 152-172, 269-313.

30. Nordenström BE (1994) The paradigm of biologically closed electric circuits (BCEC) and the formation of an International Association (IABC) for BCEC systems. Eur $J$ Surg Suppl : 7-23. [Crossref]

31. Bistolfi F (1991) Application of the order/disorder model to the interaction between non-ionizing radiation and bio structures. Radiol Med 81: 92-98. 
32. Bistolfi F (2007) Extremely low frequency pulsed magnetic fields and multiple sclerosis: effects on neurotransmission alone or also on immunomodulation. Building a working hypothesis. Neuroradiol J 20: 676-693. [Crossref]

33. Chothia C (1989) Polyhedra for helical proteins. Nature 337: 204-205. [Crossref]

34. Teller E (1982) Energia dal cielo e dalla terra. Ed. Zanichelli.

35. Adey WR (1992) Interaction mechanisms of low-level electromagnetic fields in living systems. Oxford University Press UK 1992: 44-77.

36. Adey WR (1993) Whispering between cells: electromagnetic fields and regulatory mechanisms in tissue. Frontier Perspectives 3(2): 21-25.

37. Adey WR (1988) Physiologic signaling across cell membranes and cooperative influences of extremely low frequency electromagnetic fields. Springer-Verlag: Biological Coherence and Response to External Stimuli. Herbert Frolich (ed)

38. Jacobson JI (1986) Mc2 $=\mathrm{BvL}$ coulomb: Gravitational and EM potential in dual resonance. Indian J Theoret Phys 34:231-239.

39. Jacobson JI, Yamanashi WS (1994) A possible, physical mechanism in the treatment of neurologic disorders with externally applied pico Tesla magnetic fields. Physiol Chem Phys Med NMR 26: 287-297. [Crossref]

40. Jacobson JI (1989) On the electro-magnetic nature of life. Panminerva Med 31: 151165. [Crossref]

41. Cope FW (1978) Discontinuous magnetic field effects (Barkhausen noise) in nucleic acids as evidence for room temperature organic superconduction. Physiol Chem Phys 10: 233-246. [Crossref]

42. DelGuidice E, Doglia S, Milani M (1983) Self-focusing and ponderomotive forces of coherent electric waves; a mechanism for cytoskeleton formation and dynamics. In: Frolich H, Kremen F (eds). Coherent Excitations in Biological Systems. Springer, Berlin 122-127.

43. Jacobson JI (1993) The influence of magnetism on genes. Chinese Med Sci J 8(1): $44-48$.

44. Jacobson JI (1989) On the electro-magnetic nature of life. Panminerva Med 31: 151165. [Crossref]

45. Jacobson JI (2015) Jacobson Resonance: Inertial Electromagnetic Induction. Serials Pub 2015, in press.

46. Jacobson JI (1989) The mathematical framework essential for magneto-therapy in the treatment of genomic and associated disorders, including cancer, AIDS, and CNS regeneration. Panminerva Med 31: 1-7. [Crossref]

47. Bistolfi F (1990) A hydrogen-harps model for intracellular communication and its implications for the second genetic code. Panminerva Med 32: 4-9. [Crossref]

48. Baggott J (1992) The meaning of quantum theory. Oxford Univ Press. Oxford, UK 182-183.

49. McClintock B (1931) The Order of the Genes C, Sh and Wx in Zea Mays with Reference to a Cytologically Known Point in the Chromosome. Proc Natl Acad Sci U $S A$ 17: 485-491. [Crossref]

50. Tonegawa S (1983) Somatic generation of antibody diversity. Nature 302: 575-581. [Crossref]

51. Jacobson JI (1992) A theoretical look at genes as magneto-targets. Indian $J$ Theoret Phys 40(3): 161-186.

52. Jacobson JI1 (1996) Therapeutic radiology: a potential unfolding through bioelectromagnetic sciences. Altern Ther Health Med 2: 49-55. [Crossref]

53. Levi-Montalcini R, Booker B (1960) Excessive growth of the sympathetic ganglia evoked by a protein isolated from mouse salivary glands. Proc Natl Acad Sci US A 46: 373-384. [Crossref]

54. Jacobson JI (1988) Normal and diseased states related to interdependence of electromagnetic fields, growth, repair and genetic regulation of metabolic function Indian J Biochem Biophys 25:442-6. [Crossref]

55. Jacobson JI (1990) The intrinsic electro-gravitational mechanism of life, the basis of neoplasia, and the clinical method of repair. Panminerva Med 32: 159-171. [Crossref]

56. Cohen D (1972) Magnetoencephalography: detection of the brain's electrical activity with a superconducting magnetometer. Science 175: 664-666. [Crossref]

57. Anninos PA, Tsagas N (1989) Localization and cure of epileptic foci with the use of MEG measurements. Int J Neurosci 46: 235-242. [Crossref]
58. Anninos PA, Tsagas N, Sandyk R, Derpapas K (1991) Magnetic stimulation in the treatment of partial seizures. Int J Neurosci 60: 141-171. [Crossref]

59. Anninos PA, Jacobson JI, Tsagas N, Adamopoulos A (1997) Spaciotemporal stationarity of epileptic focal activity evaluated by analyzing magnetoencephalographic (MEG) data and the theoretical implications. Panminerva Med 39(3): 189-201. [Crossref]

60. Williamson SJ, Kaufman I (1987) Analysis of neuromagnetic signals. In: Methods of Analysis of Brain Electrical and Magnetic Signals. EEG Handbook, (A.S Gevens and A Remond; eds); Revised 11: 411-417.

61. Jacobson JI, Yamanashi WS, Saxena A, Parekh P, Brown B, et al. (2000) Effect of magnetic fields on mice sciatic nerves, in-vitro. Frontier Perspectives 1: 6-11.

62. Jacobson JI, Gorman R, Yamanashi WS, Dayton M, Haltiwanger S, et al. (2001) PicoTesla range magnetic fields tested in four-site double blind clinical study for treatment of osteoarthritis knees. Alt Therap Health and Med 7: 54-69.

63. Klepitskaya O, Kumar R (2008) Efficacy and safety of low-level electromagnetic field treatment in Parkinson's disease. Movement disorders 23: 1628-37.

64. Sandyk R (1992) Successful treatment of multiple sclerosis with magnetic fields. Int J Neurosci 66: 237-250. [Crossref]

65. Jacobson JI (1988) A brief review of picoTesla range magnetotherapies. Estratto Da Gazzetta Medico Italiana-Archivo Per I.E. Sci Med 157: 37-41.

66. Trostel CT, McLaughlin RM, Lamberth JG, Cooper RC, Elder SH, et al. (2003) Effects of pico-tesla electromagnetic field treatment on wound healing in rats. Am J Vet Res 64: 845-854. [Crossref]

67. Qin C, Evans JM, Yamanashi WS, Scherlag BJ, Foreman RD (2005) Effects on rats of low intensity and frequency electromagnetic field stimulation on thoracic spinal neurons receiving noxious cardiac and esophageal inputs. Neuromod 8: 79-87. [Crossref]

68. Frolich H (1986) Coherent Excitations in active biological systems. In: Gutmann I Keyzer H(eds) Modern Bioelectrochem Plenum, NY 241-262.

69. Liboff AR, Williams T Jr, Strong DM, Wistar R Jr (1984) Time-varying magnetic fields: effect on DNA synthesis. Science 223: 818-820. [Crossref]

70. Lyle DB, Wang XH, Ayotte RD, Sheppard AR, Adey WR (1991) Calcium uptake by leukemic and normal T-lymphocytes exposed to low frequency magnetic fields. Bioelectromagnetics 12: 145-156. [Crossref]

71. Byrus CV, Kartum K, Preper S, and Adey WR (1988) Increased ornithine decarboxylase activity in cultured cells exposed to low energy modulated microwave fields and phorbol ester tumor promoters. Cancer Res 48: 4222-4226. [Crossref]

72. Goodman R, Henderson AS (1989) Exposure of salivary gland cells in low frequency electromagnetic field alters polypeptide synthesis. Proc Natl Lead Sci 1009: 216-220. [Crossref]

73. Goodman R, Weil-X, Xu J-C, Henderson AS (1989) Exposure of human cells in low frequency electromagnetic fields results in quantitative changes in transcripts. Biochem Biophys Acta 1009: 216-220. [Crossref]

74. Goodman R, Henderson AS (1990) Transcription in cells exposed to extremely low frequency electromagnetic fields: a review. Bioeletrochem Bioenerget 1990; 25:335353.

75. Delgado JM, Leal J, Monteagudo JL, Gracia MG (1982) Embryological changes induced by weak, extremely low frequency electromagnetic fields. J Anat 134: 533 551. [Crossref]

76. Jacobson JI1 (1991) The question of ameliorating the aging process, even macula regeneration with magnetic fields. Panminerva Med 33: 205-208. [Crossref]

77. Jacobson JI (1987) A testable theoretical model for the mechanism of magneto-therapy Panminerva Med 29: 263-270. [Crossref]

78. Jacobson JI (2012) Reason For Life. Abbott Press, A Division of Writers Digest Bloomington, Indiana. 117-118.

79. Costanzo LS (1998) Physiology. WB Saunders Phila 112-126.

80. Li S, Scherlag BJ, Yu L, Sheng X, Zhang Y, et al. (2009) Low-level vagosympathetic stimulation a paradox and potential new modality for the treatment of focal atria fibrillation. Circulation 2: 645-651. [Crossref]

81. Yu L, Scherlag BJ, Li S, Sheng X, Lu Z, et al. (2011) Low-level vagosympathetic nerve stimulation inhibits atrial fibrillation inducibility: direct evidence by neural recordings from intrinsic cardiac ganglia. J Cardiovasc Electrophysiol 22: 455-463. [Crossref] 
82. Sheng X, Scherlag BJ, Yu L, Li S, Ali R, et al. (2011) Prevention and reversal of atrial fibrillation inducibility and autonomic remodeling by low-level vagosympathetic nerve stimulation. J Amer Coll Cardiol 57: 563-571. [Crossref]

83. Sha Y, Scherlag BJ, Yu L, Sheng X, Jackman WM, et al. (2011) Low-level right vagal stimulation: anticholinergic and antiadrenergic effects. J Cardiovasc Electrophysiol 22: 1147-1153. [Crossref]

84. Yu L, Scherlag BJ, Sha Y, Li S, Sharma T, et al. (2012) Interactions between atrial electrical remodeling and autonomic remodeling: how to break the vicious cycle. Heart Rhythm 9: 804-809. [Crossref]

85. Smith FM1, McGuirt AS, Leger J, Armour JA, Ardell JL (2001) Effects of chronic cardiac decentralization on functional properties of canine intracardiac neurons in vitro. Am J Physiol Regul Integr Comp Physiol 281: R1474-1482. [Crossref]

86. Yu L, Scherlag BJ, Li S, Sheng X, Lu Z, et al. (2011) Low-level vagosympathetic nerve stimulation inhibits atrial fibrillation inducibility: direct evidence by neural recordings from intrinsic cardiac ganglia. J Cardiovasc Electrophysiol 22: 455-63. [Crossref]

87. Stavrakis S, Scherlag BJ, Fan Y, Liu Y, Liu Q, et al. (2012) Antiarrhythmic effects of vasostatin-1 in a canine model of atrial fibrillation. J Cardiovasc Electrophysiol 23: 771-777. [Crossref]

88. Yu L, Dyer JW, Scherlag BJ, Stavrakis S, Sha Y, et al. (2015) The use of low-level electromagnetic fields to suppress atrial fibrillation. Heart Rhythm 12: 809-817. [Crossref]

89. Kaijser L, Sachs C (1985) Autonomic cardiovascular responses in old age. Clin Physiol 5: 347-357. [Crossref]

90. Kannel WB, Wolf PA, Benjamin EJ, Levy D (1998) Prevalence, incidence, prognosis, and predisposing conditions for atrial fibrillation: population-based estimates. $\mathrm{Am} \mathrm{J}$ Cardiol 82: 2N-9N. [Crossref]

91. Einstein A (1996) The Quotable Einstein. Collected and edited by Alice Calaprice. Princeton University Press 165-185.

92. Barnett L (1974) The Universe and Dr. Einstein. Bantam Books, (7th printing) William Morrow \&Co. New York 111

93. Einstein A (956) The meaning of relativity including the relativistic theory of the nonsymmetric field. Princeton Univ Press. Princeto 1956; 165-166.

94. Einstein A (1961) Relativity: The Special and General Theory. Crown Publishers. New York 1-100

95. Einstein A (1956) Out of my later years. Citadel Press NJ 59-111.
96. Jacobson JI (1983) The secret of life. Philosophical Library, NY11-43.

97. Brancaiso P (195) The nature of physics. Macmillan Pub, NY611-659.

98. Jacobson JI (1988) Quantum phenomena proposed as mechanism for radiographic corona structures associated with pulmonary masses. Panminerva Med 30: 133-143. [Crossref]

99. Jacobson JI (1990) Is the fusion process the basis for growth, repair and aging? Panminerva Med 32: 132-140. [Crossref]

100. Jacobson JI (1992) A theoretical look at gravity in the human cell: its role in normal cell division as well as neoplasia. Panminerva Med 34: 99-106. [Crossref]

101. Coyne C, Jacobson JI (2001) Inhibition of the viability and/or proliferation rate of human mammary carcinoma cell populations (HTB-126/McF-7) with pico-Tesla range magnetic fields. From: Conference on "The effects of pico-Tesla range magnetic fields on biological systems; 2001; Mississippi State University, college of veterinary medicine.

102. Dixey R, Rein G (1982) 3H-noradrenaline release potentiated in a clonal nerve cell line by low-intensity pulsed magnetic fields. Nature 296: 253-256. [Crossref]

103. Thompsen DF (1983) Fractional Hall Effect indicates novel quantum liquid. Physics Today 19. [Crossref]

104. Jacobson JI (1996) Jacobson Resonance: The quantum mechanical basis for a nove radiological approach to treating cancer and AIDS. Frontier Perspectives 6: 17-26.

105. Volkenstein MK (1981) Simple physical presentation of enzymatic catalysis. J Theor Biol 89: 45-51. [Crossref]

106. Hooft OG (1974) Magnetic monopoles in unified gauge theory. Nuclear Phys 379: 276-284.

107. Weldrop M (1985) String as a theory of everything. Science 20: 1251.

108. Jacobson JI (2015) Jacobson Resonance: inertial electromagnetic induction. Intl J Phys 2: In Press.

109. JI (2015) Testing for electrogravitational flux quantum circuitry in biological photonphonon transduction system yields basis for inertial electromagnetic induction and the new particle-wave equation of Jacobson Resonance. OMICS, Engineering J (IER) In Press.

110. Lisi A, Ledda M, de Carlo F, Pozzi D, Messina E, et al. (2008) Ion cyclotron resonance as a tool in regenerative medicine. Electromagn Biol Med 27: 127-133. [Crossref]

Copyright: (C2016 Jacobson JI. This is an open-access article distributed under the terms of the Creative Commons Attribution License, which permits unrestricted use, distribution, and reproduction in any medium, provided the original author and source are credited. 